\title{
ON THE COHOMOLOGY OF LINEAR GROUPS OVER IMAGINARY QUADRATIC FIELDS
}

\author{
MATHIEU DUTOUR SIKIRIĆ, HERBERT GANGL, PAUL E. GUNNELLS, JONATHAN HANKE, \\ ACHILL SCHÜRMANN, AND DAN YASAKI
}

\begin{abstract}
Let $\Gamma$ be the group $\operatorname{GL}_{N}\left(\mathcal{O}_{D}\right)$, where $\mathcal{O}_{D}$ is the ring of integers in the imaginary quadratic field with discriminant $D<0$. In this paper we investigate the cohomology of $\Gamma$ for $N=3,4$ and for a selection of discriminants: $D \geq-24$ when $N=3$, and $D=-3,-4$ when $N=4$. In particular we compute the integral cohomology of $\Gamma$ up to $p$-power torsion for small primes $p$. Our main tool is the polyhedral reduction theory for $\Gamma$ developed by Ash [4, Ch. II] and Koecher [24. Our results extend work of Staffeldt [40, who treated the case $N=3, D=-4$. In a sequel [15] to this paper, we will apply some of these results to computations with the $K$-groups $K_{4}\left(\mathcal{O}_{D}\right)$, when $D=-3,-4$.
\end{abstract}

\section{INTRODUCTION}

1.1. Let $F$ be an imaginary quadratic field of discriminant $D<0$, let $\mathcal{O}=\mathcal{O}_{D}$ be its ring of integers, and let $\Gamma$ be the group $\mathrm{GL}_{N}(\mathcal{O})$. The homology and cohomology of $\Gamma$ when $N=2$ - or rather its close cousin the Bianchi group $\operatorname{PSL}_{2}(\mathcal{O})$ - have been well studied in the literature. For an (incomplete) selection of results we refer to [5, 13, 28, 29, 33, 34, 44. Today we have a good understanding of a wide range of examples, and one can even compute them for very large discriminants (cf. [46]). For $N>2$, on the other hand, the group $\Gamma$ has not received the same attention. The first example known to us is the work of Staffeldt [40]. He treated the case $N=3, D=-4$ with the goal of understanding the 3 -torsion in $K_{3}(\mathbb{Z}[\sqrt{-1}])$. The second example is [10], which investigates the case of the groups $\operatorname{GL}(L)$ for $L$ an $\mathcal{O}$-lattice that is not necessarily a free $\mathcal{O}$-module. This allows the authors to compute the Hermite constants of those rings in case $D \geq-10$ and $\operatorname{rank}(L) \leq 3$. Our methods apply as well to the non-free case, and the corresponding cohomology computations should be useful when investigating automorphic forms over number fields that are not principal ideal domains (cf. [41, Appendix] for more about the connection between cohomology of arithmetic groups and automorphic forms).

In this paper we rectify this situation somewhat by beginning the first systematic computations for higher rank linear groups over $\mathcal{O}$. In particular we investigate the cohomology of $\Gamma$ for $N=3,4$ and for a selection of discriminants: $D \geq-24$ when $N=3$, and $D=-3,-4$ when $N=4$. We explicitly compute the polyhedral reduction domains arising from Voronoi's theory of perfect forms, as generalized by Ash [4, Ch. II] and Koecher 24. This allows us

2010 Mathematics Subject Classification. Primary 11F75; Secondary 11F67, 20J06.

Key words and phrases. Cohomology of arithmetic groups, Voronoi reduction theory, linear groups over imaginary quadratic fields.

MDS was partially supported by the Croatian Ministry of Science, Education and Sport under contract 098-0982705-2707 and by the Humboldt Foundation. PG was partially supported by the NSF under contract DMS 1101640. JH was partially supported by the NSF under contract DMS-0603976. The authors thank the American Institute of Mathematics, where this research was initiated. 
to compute the integral cohomology of $\Gamma$ up to $p$-power torsion for small primes $p$. In a sequel [15] to this paper, we will apply some of these results to computations with the $K$-groups $K_{4}\left(\mathcal{O}_{D}\right)$, when $D=-3,-4$.

1.2. Here is a guide to the paper (which closely follows the structure of the first five sections of [19]). In Section 2 we recall the explicit reduction theory we need to build our chain complexes to compute cohomology. In Section 3 we define the complexes, and explain the relation between what we compute and the cohomology of $\Gamma$. In Section 4 we describe a "mass formula" for the cells in our tessellations that provides a strong computational check on the correctness of our constructions. In Section 5 we give an explicit representative for the non-trivial class in the top cohomological degree; this construction is motivated by a similar construction in [18, 19]. The algorithms we used are described in Section 6. Finally, in Section 7 we give the results of our computations.

1.3. Acknowledgments. We thank A. Ash, Ph. Elbaz-Vincent, and C. Soulé for helpful discussions. We thank an anonymous referee for suggesting improvements to our paper. This research, and the research in the companion papers [15, 16] was conducted as part of a "SQuaRE" (Structured Quartet Research Ensemble) at the American Institute of Mathematics in Palo Alto, California in February 2012. It is a pleasure to thank AIM and its staff for their support, without which our collaboration would not have been possible. Also our initial computations of cohomology and $K$-groups occurred on the large shared computers parsley and rosemary provided to $\mathrm{JH}$ at the University of Georgia mathematics department, and we thank them for their support in allowing these machines to be used for collaborative mathematical research projects.

\section{The POLYHEDRAL CONES}

Fix $N>0$ and an imaginary quadratic field $F$ of discriminant $D<0$ with ring of integers $\mathcal{O}=\mathcal{O}_{D}$ and define the element

$$
\omega:=\omega_{D}:= \begin{cases}\sqrt{D / 4} & \text { if } D \equiv 0 \bmod 4, \\ (1+\sqrt{D}) / 2 & \text { if } D \equiv 1 \bmod 4,\end{cases}
$$

so that $F=\mathbb{Q}(\omega)$ and $\mathcal{O}=\mathbb{Z}[\omega]$. Throughout we fix a complex embedding $F \hookrightarrow \mathbb{C}$ and consistently identify $F$ with its image in $\mathbb{C}$. We also extend this identification to vectors and matrices with coefficients in $F$. Furthermore, we put $\Gamma=\mathrm{GL}_{N}(\mathcal{O})$.

2.1. Hermitian forms. Let $\mathcal{H}^{N}(\mathbb{C})$ denote the $N^{2}$-dimensional real vector space of $N \times N$ Hermitian matrices with complex coefficients. Using the chosen complex embedding of $F$ we can view $\mathcal{H}^{N}(F)$, the Hermitian matrices with coefficients in $F$, as a subset of $\mathcal{H}^{N}(\mathbb{C})$. Moreover, this embedding allows us to view $\mathcal{H}^{N}(\mathbb{C})$ as a $\mathbb{Q}$-vector space such that the rational points of $\mathcal{H}^{N}(\mathbb{C})$ are exactly $\mathcal{H}^{N}(F)$.

Define a map $q: \mathcal{O}^{N} \rightarrow \mathcal{H}^{N}(F)$ by the outer product $q(x)=x x^{*}$, where $*$ denotes conjugate transpose (with conjugation being the non-trivial complex conjugation automorphism of $F)$. Each $A \in \mathcal{H}^{N}(\mathbb{C})$ defines a Hermitian form $A[x]$ on $\mathbb{C}^{N}$ by the rule

$$
A[x]:=x^{*} A x, \quad \text { for } x \in \mathbb{C}^{N} .
$$

Define the non-degenerate bilinear pairing

$$
\langle\cdot, \cdot\rangle: \mathcal{H}^{N}(\mathbb{C}) \times \mathcal{H}^{N}(\mathbb{C}) \rightarrow \mathbb{C}
$$


by $\langle A, B\rangle:=\operatorname{Tr}(A B)$. For $v \in \mathcal{O}^{N}$ (identified with its image in $\mathbb{C}^{N}$ ) one can easily verify that

$$
A[v]=\operatorname{Tr}(A q(v))=\langle A, q(v)\rangle .
$$

Let $C_{N} \subset \mathcal{H}^{N}(\mathbb{C})$ denote the cone of positive definite Hermitian matrices.

Definition 2.1. For $A \in C_{N}$, we define the minimum of $A$ as

$$
\min (A):=\min _{D}(A):=\inf _{x \in \mathcal{O}^{N} \backslash\{0\}} A[x] .
$$

Note that $\min (A)>0$ since $A$ is positive definite. A vector $v \in \mathcal{O}^{N}$ is called a minimal vector of $A$ if $A[v]=\min (A)$. We denote the set of minimal vectors of $A$ by $\operatorname{Min}(A)$.

It should be emphasized that these notions depend on the fixed choice of the imaginary quadratic field $F$. Since $q\left(\mathcal{O}^{N}\right)$ is discrete in $\mathcal{H}^{N}(\mathbb{C})$ and the level sets $\{q(x) \mid\langle A, q(x)\rangle=C\}$ are compact, the minimum for each $A$ is attained by only finitely many minimal vectors.

From (2), we see that each vector $v \in \mathcal{O}^{N}$ gives rise to a linear functional $q(v)$ on $\mathcal{H}^{N}(\mathbb{C})$, which acts by $A \mapsto\langle A, q(v)\rangle$.

Definition 2.2. We say a Hermitian form $A \in C_{N}$ is a perfect Hermitian form over $F$ if

$$
\operatorname{span}_{\mathbb{R}}\{q(v) \mid v \in \operatorname{Min}(A)\}=\mathcal{H}^{N}(\mathbb{C}) .
$$

From Definition 2.2 it is clear that a form is perfect if and only if it is uniquely determined by its minimum and its minimal vectors. Equivalently, a form $A$ is perfect when $\operatorname{Min}(A)$ determines $A$ up to a positive real scalar. It is convenient to normalize a perfect form by requiring that $\min (A)=1$, and we will do so throughout this paper. A priori there is no reason to expect that every perfect form (up to rescaling) can be realized as an $F$-rational point in $\mathcal{H}^{n}(\mathbb{C})$; indeed, when one generalizes these concepts to general number fields this is too much to expect (cf. [20]). However, for imaginary quadratic fields this rationality property does hold:

Theorem 2.3. 26, Theorem 3.2] Suppose $F$ is a CM field. If $A \in C_{N}$ is a perfect Hermitian form over $F$ such that $\min (A)=1$, then $A \in \mathcal{H}^{N}(F)$.

We now construct a partial compactification of the cone $C_{N}$.

Definition 2.4. A matrix $A \in \mathcal{H}^{N}(\mathbb{C})$ is said to have an $F$-rational kernel when the kernel of $A$ is spanned by vectors in $F^{N} \subset \mathbb{C}^{N}$. Let $C_{N}^{*} \subset \mathcal{H}^{N}(\mathbb{C})$ denote the subset of non-zero positive semi-definite Hermitian forms with $F$-rational kernel.

Note that $C_{N} \subset C_{N}^{*}$, since any $A \in C_{N}$ has trivial kernel, which is clearly $F$-rational.

We now describe the connection between the cones $C_{N} \subset C_{N}^{*}$ and symmetric spaces. Let $\mathbf{G}$ be the reductive group over $\mathbb{Q}$ given by the restriction of scalars $\operatorname{Res}_{F / \mathbb{Q}}\left(\mathrm{GL}_{N}\right)$. Thus $\mathbf{G}(\mathbb{Q})=\mathrm{GL}_{N}(F)$ and $\mathbf{G}(\mathbb{Z})=\Gamma=\mathrm{GL}_{N}(\mathcal{O})$. The group $\mathbf{G}(\mathbb{R})=\mathrm{GL}_{N}(\mathbb{C})$ acts on $C_{N}^{*}$ on the left by

$$
g \cdot A=g A g^{*},
$$

where $g \in \mathrm{GL}_{N}(\mathbb{C})$ and $A \in C_{N}^{*}$; one can easily verify that this action preserves $C_{N}$. Let $H=R_{d} \mathbf{G}(\mathbb{R})^{0}$ be the identity component of the group of real points of the split radical of $\mathbf{G}$. Then $H \simeq \mathbb{R}_{+}$, and as a subgroup of $\mathbf{G}(\mathbb{R})$, it acts on $C_{N}^{*}$ by positive real homotheties. Voronoi's work [45, generalized by Ash [4, Ch. II] and Koecher [24], shows that there are only finitely many perfect Hermitian forms over $F$ modulo the actions of $\Gamma$ and $H$.

Let $X_{N}^{*}$ denote the quotient $X_{N}^{*}=H \backslash C_{N}^{*}$, and let $\pi: C_{N}^{*} \rightarrow X_{N}^{*}$ denote the projection. Then $X_{N}=\pi\left(C_{N}\right)$ can be identified with the global Riemannian symmetric space for the 
reductive group $H \backslash \mathrm{GL}_{N}\left(\mathbb{C}\right.$ ), or (if one prefers) for the semisimple group $\mathrm{SL}_{N}(\mathbb{C}$ ); in terms of the latter we have $X_{N} \simeq \mathrm{SL}_{N}(\mathbb{C}) / \mathrm{SU}(N)$.

Moreover, the image $X_{N}^{*}$ of $C_{N}^{*}$ under homotheties is a partial compactification of the symmetric space $X_{N}$ and is an example of a Satake compactification [30]. The quotient $\Gamma \backslash X_{N}^{*}$ is compact with finitely many boundary components. More precisely, let $\mathfrak{g}$ be the Lie algebra of $\mathrm{SL}_{N}(\mathbb{C})$. Satake defined a collection of partial compactifications of $X_{N}$ indexed by dominant weights $\theta$ of representations of $\mathfrak{g}$. In this collection there are only finitely many topological types: the type of the compactification indexed by $\theta$ depends on which walls of the dominant chamber contain $\theta$, and the less regular the weight, the more singular the compactification. For our example $\theta$ is the first fundamental weight, and thus the quotient $\Gamma \backslash X_{N}^{*}$ is highly singular (in fact, as singular as possible in this family of compactifications). Indeed, it is a minimal Satake compactification, and in a sense is the analogue of the BailyBorel compactification (although the latter is not defined for $X_{N}$, since $X_{N}$ is not a Hermitian symmetric space). In the quotient $\Gamma \backslash X_{N}^{*}$ there are boundary components of dimensions $m^{2}-1$ for $m=1, \ldots, N-1$; the components of dimension $m^{2}-1$ are isomorphic to quotients of the symmetric space $X_{m}$ for $\mathrm{SL}_{m}(\mathbb{C})$, i.e. the symmetric space attached to the Levi factor of the standard parabolic subgroup of type $(m, 1, \ldots, 1)$. For more details about the geometry and topology of compactifications of locally symmetric spaces, we refer to 8 .

Remark 2.5. The action of $\mathbf{G}(\mathbb{R})=\mathrm{GL}_{N}(\mathbb{C})$ on $\mathcal{H}^{N}(\mathbb{C})$ gives a representation $\rho: \mathrm{GL}_{N}(\mathbb{C}) \rightarrow$ $\mathrm{GL}_{N^{2}}(\mathbb{R})$. Any element $g$ acts on the orientation of $\mathcal{H}^{N}(\mathbb{C})$ via the sign of $\operatorname{det}(\rho(g))$. Since $\mathrm{GL}_{N}(\mathbb{C})$ is connected, $\mathrm{GL}_{N}(\mathbb{C})$ and its subgroup $\mathrm{GL}_{N}(F)$ act on $\mathcal{H}^{N}(\mathbb{C})$ via orientation preserving automorphisms.

2.2. Two cell complexes. Let $M$ be a finite subset of $\mathcal{O}^{N} \backslash\{0\}$. The cone generated by $M$ is the set of non-zero matrices of the form $\sum_{v \in M} \lambda_{v} q(v)$, where $\lambda_{v} \in \mathbb{R}_{\geq 0}$. For a perfect form $A$, we call the cone $\sigma(A)$ generated by the minimal vectors of $A$ the perfect cone attached to $A$; by abuse of notation we denote the image of $\sigma(A)$ under $\pi$, which is a cell, also by $\sigma(A)$. One can show 4,24] that the cells $\sigma(A)$ and their intersections, as $A$ runs over equivalence classes of perfect forms, define a $\mathrm{GL}_{N}(\mathcal{O})$-invariant cell decomposition of $X_{N}^{*}$, with finitely many equivalence classes of cells modulo $\mathrm{GL}_{N}(\mathcal{O})$. We can make the $\mathrm{GL}_{N}(\mathcal{O})$-action explicit: for a perfect form $A \in C_{N}$ and an element $\gamma \in \mathrm{GL}_{N}(\mathcal{O})$, we have

$$
\begin{aligned}
\gamma \cdot \operatorname{Min}(A) & =\left\{\gamma \cdot v \in \mathcal{O}^{N} \mid A[v]=\min (A)\right\} \\
& =\left\{w \in \mathcal{O}^{N} \mid A\left[\gamma^{-1} w\right]=\min (A)\right\}=\operatorname{Min}\left(\left(\gamma^{*}\right)^{-1} A \gamma^{-1}\right)
\end{aligned}
$$

and therefore

$$
\gamma \cdot \sigma(A)=\text { perfect cone of }\{\gamma v \mid v \in \operatorname{Min}(A)\}=\sigma\left(\left(\gamma^{*}\right)^{-1} A \gamma^{-1}\right) .
$$

We endow $X_{N}^{*}$ with the CW-topology coming from the images of the $\sigma(A)$ and their faces [22, Appendix].

If $\tau$ is a closed cell in $X_{N}^{*}$ and $A$ is a perfect form with $\tau \subset \sigma(A)$, we let $\operatorname{Min}(\tau)$ denote the set of vectors $v \in \operatorname{Min}(A)$ such that $q(v) \in \tau$. The set $\operatorname{Min}(\tau)$ is independent of the (possible) choice of $A$. Then $\tau$ is the image in $X_{N}^{*}$ of the cone $C_{\tau}$ generated by $\{q(v) \mid v \in \operatorname{Min}(\tau)\}$, that is, $C_{\tau}=\left\{\sum_{v \in M(\tau)} \lambda_{v} q(v) \mid \lambda_{v} \in \mathbb{R}_{\geq 0}\right\}$. For any two closed cells $\tau$ and $\tau^{\prime}$ in $X_{N}^{*}$, we have $\operatorname{Min}(\tau) \cap \operatorname{Min}\left(\tau^{\prime}\right)=\operatorname{Min}\left(\tau \cap \tau^{\prime}\right)$.

Let $\tilde{\Sigma} \subset C_{N}^{*}$ be the (infinite) union of all cones $C_{\sigma}$ such that $\sigma \in X_{N}^{*}$ has non-trivial intersection with $X_{N}$. One can verify that the stabilizer of $C_{\sigma}$ in $\operatorname{GL}_{N}(\mathcal{O})$ is equal to the stabilizer of $\sigma$ in $\mathrm{GL}_{N}(\mathcal{O})$. By abuse of notation, we write $\operatorname{Min}\left(C_{\sigma}\right)$ as $\operatorname{Min}(\sigma)$. 


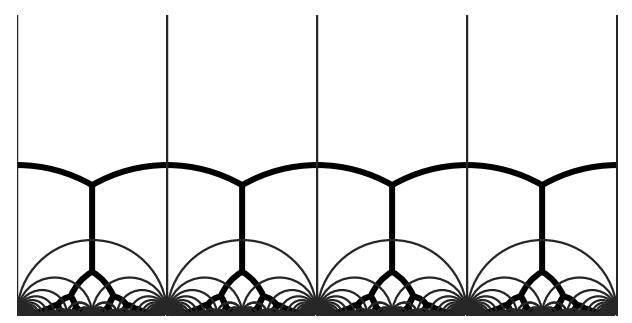

FIGURE 1. The ideal triangles of the Voronoi tessellation of the upper halfplane $\mathfrak{H}$ shown in grey thin lines, with its dual the well-rounded retract shown in heavy black lines.

We now recall the well-rounded retract of Ash 2, a contractible $\left(N^{2}-N\right)$-dimensional cell complex $W$ on which $\mathrm{GL}_{N}(\mathcal{O})$ acts with finite stabilizers. A non-zero finite set $M \subset \mathcal{O}^{N}$ is called well-rounded if the $\mathbb{C}$-span of $M$ is $\mathbb{C}^{N}$. For a well-rounded subset $M$, let $\mu(M)$ denote the set of forms $A \in C_{N}$ with $\operatorname{Min}(A)=M$ and $\min (A)=1$. It is easy to prove that if $\mu(M)$ is non-empty then it is convex and thus topologically a cell. The well-rounded retract is then defined to be

$$
W=\bigcup_{M \text { well-rounded }} \mu(M) .
$$

The space $W$ is dual to the decomposition of $X_{N}$ induced by the cones $\tilde{\Sigma}$ (see Theorem 2.7 below). Figure 1 shows the relationship between $W$ and the cells induced by $\tilde{\Sigma}$ when $N=2$ and $F=\mathbb{Q}$. In this case $X$ can be identified with the upper half-plane $\mathfrak{H}=\{x+i y \mid y>0\}$. The Voronoi tessellation is the tiling of $\mathfrak{H}$ by the $\mathrm{SL}_{2}(\mathbb{Z})$-translates of the ideal geodesic triangle with vertices $\{0,1, \infty\}$. The well-rounded retract $W$ is the dual infinite trivalent tree.

Lemma 2.6. If $C_{\sigma} \in \tilde{\Sigma}$, then $\operatorname{Min}(\sigma)$ is well-rounded.

Proof. This is proved by McConnell in [25, Theorem 2.11] when $F=\mathbb{Q}$, and we mimic his proof to yield the analogous result for imaginary quadratic fields. Let $M=\operatorname{Min}\left(C_{\sigma}\right)$ denote the spanning vectors of $C_{\sigma}$. Suppose $M$ is not well-rounded. Then $M$ does not span $\mathbb{C}^{N}$, and so there exists a non-zero vector $w \in \mathbb{C}^{N}$ such that $v w^{*}=0$ for all $v \in M$. Then each $\theta \in C_{\sigma}$ can be written as a non-zero Hermitian form $\theta=\sum_{v \in M} a_{v} q(v)$, where $a_{v} \geq 0$. It follows that

$$
\theta[w]=\sum_{v \in M} a_{v}\langle q(v), q(w)\rangle=\sum_{v \in M} a_{v}\left|v w^{*}\right|^{2}=0,
$$

where $|\cdot|$ is the usual norm on $\mathbb{C}^{N}$. In particular, $\theta$ is not positive definite, contradicting the assumption that $C_{\sigma} \in \tilde{\Sigma}$.

Theorem 2.7. Let $M \subset \mathcal{O}^{N}$ be well-rounded and let $\mu(M) \subset W$ be non-empty. Then there is a unique cone $C_{\sigma} \in \tilde{\Sigma}$ such that $\operatorname{Min}\left(C_{\sigma}\right)=M$. The map $\mu(M) \mapsto C_{\sigma}$ is a canonical bijection $W \rightarrow \tilde{\Sigma}$ that is inclusion-reversing on the face relations.

Proof. It suffices to prove that a well-rounded subset $M \subset \mathcal{O}^{N}$ has $\mu(M) \neq \emptyset$ if and only if there is a cone $C_{\sigma} \in \tilde{\Sigma}$ such that $\operatorname{Min}(\sigma)=M$.

Suppose $C_{\sigma} \in \tilde{\Sigma}$. By Lemma 2.6, $M=\operatorname{Min}\left(C_{\sigma}\right)$ is well-rounded. Thus it remains to show that $\mu(M)$ is non-empty. We do so by constructing a form $B \in \mu(M)$ with $\operatorname{Min}(B)=$ $\operatorname{Min}\left(C_{\sigma}\right)$. Since $C_{\sigma} \in \tilde{\Sigma}$, there is a perfect form $A$ such that $C_{\sigma}$ is a face of the cone 
$S_{A}=\pi^{-1}(\sigma(A))$. Furthermore, $C_{\sigma}$ can be described as the intersection of $S_{A}$ with some supporting hyperplane $\{\theta \mid\langle H, \theta\rangle=0\}$ for some $H \in \mathcal{H}^{N}(\mathbb{C})$. It follows that

$$
\langle H, q(v)\rangle=0 \quad \text { if } v \in M \quad \text { and } \quad\langle H, q(v)\rangle>0 \quad \text { if } v \in \operatorname{Min}(A) \backslash M .
$$

Let $B=A+\rho H$. Since $\langle H, q(v)\rangle=B[v]$, a standard argument for Hermitian forms shows that for sufficiently small positive $\rho, B$ is positive definite, $B[v]=\min (A)$ for $v \in M$ and $B[v]>\min (A)$ for $v \in \operatorname{Min}(A) \backslash M$. Thus $\operatorname{Min}(B)=M$ and so $B \in \mu(M)$. In particular, $\mu(M)$ is non-empty.

Conversely, suppose $M$ is a well-rounded subset of $\mathcal{O}^{N}$ with $\mu(M)$ non-empty. Choose $B \in \mu(M)$ so that $\operatorname{Min}(B)=M$. If $B$ is perfect, then $M=\operatorname{Min}(B)$ and we are done. Otherwise, we can use a generalization of an algorithm of Voronoi [16] to find a perfect form $A$ such that $\operatorname{Min}(B) \subsetneq \operatorname{Min}(A)$. Let $H=A-B$. Then

$$
\langle H, q(v)\rangle=0 \quad \text { if } v \in M \quad \text { and } \quad\langle H, q(v)\rangle>0 \quad \text { if } v \in \operatorname{Min}(A) \backslash M .
$$

Thus the hyperplane $\{\theta \mid\langle H, \theta\rangle=0\}$ is a supporting hyperplane for the subset of $S_{A}=$ $\pi^{-1}(\sigma(A))$ spanned by $\{q(v) \mid v \in M\}$. Therefore $M$ defines a face of $S_{A}$ in $\tilde{\Sigma}$ as desired.

Definition 2.8. Let $\Sigma_{n}^{*}$ denote a set of representatives, modulo the action of $\mathrm{GL}_{N}(\mathcal{O})$, of $n$-dimensional cells of $X_{N}^{*}$ that meet $X_{N}$. Let $\Sigma^{*}=\cup_{n} \Sigma_{n}^{*}$. The cells that are quotients of cones not meeting $C_{N}$ are said to be at infinity.

We note that $\pi(\tilde{\Sigma})=\mathrm{GL}_{N}(\mathcal{O}) \cdot \Sigma^{*}$. In the following corollary we explain why we can work with $\Sigma^{*}$ and its stabilizer subgroups as if we were working with the proper, contractible $\mathrm{GL}_{N}(\mathcal{O})$-complex $W$.

Corollary 2.9. The map $W \rightarrow \tilde{\Sigma}$ of Theorem 2.7 is a $\mathrm{GL}_{N}(\mathcal{O})$-equivariant bijection.

Proof. To see this, let $\mu(M)$ be a cell in $W$. Then the forms $B \in \mu(M)$ have $\operatorname{Min}(B)=M$ and $\min (B)=1$. Under the identification in Theorem 2.7 $\mu(M)$ corresponds to the cone $C_{\sigma}^{\prime}$ with spanning vectors $\{q(v) \mid v \in M\}$. Let $\sigma^{\prime}$ be the corresponding cell in $X_{N}^{*}$. There is a cell $\sigma \in \Sigma^{*}$ that is $\mathrm{GL}_{N}(\mathcal{O})$-equivalent to $\sigma^{\prime}$. If $\gamma \in \mathrm{GL}_{N}(\mathcal{O})$, then $\gamma \cdot \mu(M)=$ $\mu\left(\left\{\left(\gamma^{*}\right)^{-1} v \mid v \in M\right\}\right)$. Thus if $\gamma \in \operatorname{Stab}(\mu(M))$, then $\left(\gamma^{*}\right)^{-1} \in \operatorname{Stab}\left(C_{\sigma}^{\prime}\right)$. It is therefore clear that $\operatorname{Stab}\left(C_{\sigma}^{\prime}\right)=\operatorname{Stab}\left(\sigma^{\prime}\right) \simeq \operatorname{Stab}(\sigma) \simeq \operatorname{Stab}(\mu(M))$.

The map $g \mapsto\left(g^{*}\right)^{-1}$ is an isomorphism of groups, so the duality between $W \bmod \Gamma=$ $\mathrm{GL}_{N}(\mathcal{O})$ and $\Sigma^{*}$ in Theorem 2.7 allows us to work with $\Sigma^{*}$ and its stabilizer subgroups as if we were working with a proper, contractible $\mathrm{GL}_{N}(\mathcal{O})$-complex. This fact will be used later to compute the mass formula (\$4).

2.3. An example. We consider the Eisenstein integers $\mathcal{O}=\mathbb{Z}[\omega]$, where $\omega=\frac{1+\sqrt{-3}}{2}$, and give the details of the $\Sigma^{*}$ computation for $\Gamma=\mathrm{GL}_{3}(\mathcal{O})$. In particular, we give representatives of the $\mathrm{GL}_{3}(\mathcal{O})$-equivalence classes of cells in $X_{3}^{*}$ and describe the facets of each cell. This includes not only $\mathrm{GL}_{3}(\mathcal{O})$-representatives for $\pi(\tilde{\Sigma})$, but also representatives for the cells at infinity.

Let $A$ denote the matrix

$$
A=\left[\begin{array}{ccccccccccccc}
1 & 1 & 1 & 1 & 1 & 1 & 0 & 0 & 0 & 1 & 1 & 1 & 0 \\
-\omega-1 & -\omega-1 & -\omega & -1 & -\omega & -1 & 1 & 1 & 0 & -\omega & -1 & 0 & 1 \\
1 & \omega & 0 & 0 & \omega & 1 & -1 & 0 & 1 & \omega^{2} & \omega & 0 & -\omega
\end{array}\right]
$$

We describe $\Sigma^{*}$ in terms of the columns of $A$. In what follows, we denote by a list of integers $\left[m_{1}, \ldots, m_{k}\right]$ the image in $X_{3}^{*}$ of the cone generated by the columns of $A$ indexed by $m_{1}, \ldots, m_{k}$. 
There are two equivalence classes of perfect Hermitian forms modulo the $\Gamma$-action, so the 8-dimensional cells in $\Sigma^{*}$ fall into two orbits, with representatives

$$
a_{1}=[1,2,3,4,5,6,7,8,9] \quad \text { and } \quad a_{2}=[2,3,4,5,6,7,8,9,10,11,12,13] .
$$

Recall that the $f$-vector of a $d$-dimensional polytope $\sigma$ is the vector $\left(f_{-1}, f_{0}, f_{1}, \ldots, f_{d}\right)$, where $f_{k}$ is the number of $k$-dimensional faces of $\sigma$ and $f_{-1}:=f_{d}$. The $f$-vector of $a_{1}$ is

$$
f\left(a_{1}\right)=(1,9,36,84,126,126,84,36,9,1) .
$$

Since $a_{1}$ has nine vertices in an 8-dimensional space, we see that $a_{1}$ is a simplex, and this is indeed compatible with its $f$-vector. As such, the faces of $a_{1}$ are easily described as all of the subsets of $\{1,2, \ldots, 9\}$. The cone $a_{2}$ is not a simplex, but it is a simplicial polytope since all of its 81 facets are simplices. The $f$-vector of $a_{2}$ is

$$
f\left(a_{2}\right)=(1,12,66,216,459,648,594,324,81,1) .
$$

For simplicial polytopes, one often prefers to use the (simpler) $h$-vector, which is related to the $f$-vector via the transformation

$$
\sum_{i=0}^{d} f_{i-1}(t-1)^{d-i}=\sum_{k=0}^{d} h_{k} t^{d-k} .
$$

Here, $t$ is a formal variable that just serves to access the binomial coefficients. Using this, one finds that the $h$-vector of $a_{2}$ is

$$
h\left(a_{2}\right)=(1,4,10,16,19,16,10,4,1) .
$$

The 7-dimensional cells in $\Sigma^{*}$ fall into two equivalence classes under the action of $\Gamma$, namely

$$
b_{1}=[1,2,3,4,5,6,7,8] \text { and } b_{2}=[4,6,7,8,9,11,12,13] ;
$$

these are both simplicial. All nine of the facets of $a_{1}$ are of type $b_{1}$. Of the 81 facets of $a_{2}$, nine are of type $b_{1}$ and 72 are of type $b_{2}$.

The 6-dimensional cells in $\Sigma^{*}$ fall into three equivalence classes

$$
c_{1}=[1,2,3,4,5,6,7], \quad c_{2}=[4,6,8,9,11,12,13], \quad \text { and } \quad c_{3}=[4,6,7,8,9,11,13] .
$$

The eight facets of $b_{1}$ are type $c_{1}$. There are six facets of $b_{2}$ of type $c_{2}$ and one each of types $c_{1}$ and $c_{3}$.

The 5-dimensional cells of $\Sigma^{*}$ fall into four equivalence classes

$$
\begin{array}{ll}
d_{1}=[1,2,3,4,5,6], & d_{2}=[1,2,3,4,5,7], \\
d_{3}=[4,6,8,9,11,12], & d_{4}=[4,8,9,11,12,13] .
\end{array}
$$

The cell $c_{1}$ has one facet of type $d_{1}$ and six facets of type $d_{2}$. The cell $c_{2}$ has three facets of type $d_{2}$, three facets of type $d_{3}$, and one facet of type $d_{4}$. The cell $c_{3}$ has one facet of type $d_{1}$ and three facets of type $d_{3}$.

The 4-dimensional cells of $\Sigma^{*}$ fall into three equivalence classes

$$
e_{1}=[1,2,3,4,5], \quad e_{2}=[1,2,3,4,7], \quad \text { and } \quad e_{3}=[4,6,8,9,11] .
$$

All six of the facets of $d_{1}$ are type $e_{1}$. The cell $d_{2}$ has three facets of type $e_{1}$ and $e_{2}$ each. The cell $d_{3}$ has one facet of type $e_{1}$, three facets of type $e_{2}$, and two facets of type $e_{3}$. All six of the facets of $d_{4}$ are type $e_{2}$.

The 3-dimensional cells of $\Sigma^{*}$ fall into two equivalence classes

$$
f_{1}=[1,2,3,4] \text { and } f_{2}=[1,2,4,5] .
$$


The cell $e_{1}$ has three facets of type $f_{1}$ and two facets of type $f_{2}$. The cell $e_{2}$ has one facet of type $e_{1}$ and four facets of type $f_{2}$. The cell $e_{3}$ has one facet at infinity and four facets of type $f_{2}$.

The 2-dimensional cells of $\Sigma^{*}$ fall into a single equivalence class

$$
g_{1}=[1,2,3]
$$

All of the facets of $f_{1}$ are type $g_{1}$. The cell $f_{2}$ has one facet at infinity and three facets of type $g_{1}$. The boundary of $g_{1}$ consists of three 1-cells. Note that since we are considering an $N=3$ example, every cone generated by two minimal vectors is necessarily at infinity. In particular, $g_{1}$ has all of its boundary edges at infinity, but is not itself at infinity. An analogous situation occurs in the Voronoi tessellation of the upper half-plane shown in Figure 1 where we have a 1-cell whose boundary vertices are at infinity, but is not itself at infinity.

We now consider the cells in $\Sigma^{*}$ and their relationship with boundary components in the Satake compactification $X_{3}^{*}$. The symmetric space $X_{3}$ has dimension 8 , and the compactification $X_{3}^{*}$ has boundary components of dimensions 3 and 0 . The former are isomorphic to hyperbolic 3 -space $\mathfrak{H}_{3}$, and the latter are of course points.

The cells that lie at infinity in the 3-dimensional boundary components are certain facets in the boundaries of three equivalence classes above, represented by the 4 -cell $e_{3}$, the 3 -cell $f_{2}$, and the 2-cell $g_{1}$. The cell $e_{3}$ has one boundary facet at infinity, $f_{2}$ has one boundary facet at infinity, and $g_{1}$ has all its boundary facets at infinity. These boundary faces have dimensions 3, 2, 1 respectively, and all cells of a given dimension are $\mathrm{GL}_{3}(\mathcal{O})$-equivalent. Taken together they form ideal polyhedra that tessellate the hyperbolic 3-spaces.

More precisely, fix a boundary component $X_{2}$ of dimension 3 and identify it with $\mathfrak{H}_{3}$. Then the cells at infinity meeting $X_{2}$ tessellate $X_{2} \simeq \mathfrak{H}_{3}$ exactly as in Cremona's study of modular symbols for imaginary quadratic fields in the case $\mathbb{Q}(\sqrt{-3})[13$. To construct this hyperbolic tessellation, one takes the ideal tetrahedron in $\mathfrak{H}_{3}$ with vertices at $0,1, \omega$, and $\infty$, and translates it by $\mathrm{GL}_{2}(\mathcal{O})$ (cf. [13, Fig. 2.3.3]). In particular all the dimension 3 boundary components inherit a tessellation by ideal tetrahedra isomorphic to this one. Indeed, one can check that the cell appearing in the boundary of $e_{3}$ indexed by $[4,6,9,11]$ is the one at infinity, and that mod $\mathrm{GL}_{3}(\mathcal{O})$ this set of minimal vectors can be taken to the columns of

$$
\left[\begin{array}{llll}
\omega & 0 & 1 & 1 \\
1 & 1 & 1 & 0 \\
0 & 0 & 0 & 0
\end{array}\right],
$$

which give the ideal vertices of the tessellation of the standard boundary component of dimension 3 corresponding to the standard maximal parabolic subgroup of $\mathrm{SL}_{3}(\mathbb{C})$ of type $(2,1)$. The $\mathrm{GL}_{3}(\mathcal{O})$-action transports this tessellation to the other boundary components.

This almost accounts for all the cells at infinity. The remaining cells at infinity are points appearing in the boundaries of the 1-dimensional cells. As one might expect, these points are nothing more than the boundary components of dimension 0 ; they lie in a single $\mathrm{GL}_{3}(\mathcal{O})$ orbit. In terms of the hyperbolic 3-space picture, these points are exactly the ideal vertices of the ideal polyhedra tessellating the $\mathfrak{H}_{3}$ 's. This reflects the inductive nature of Satake's construction: inside $X_{3}^{*}$, any 3-dimensional boundary component $X_{2}$ inherits a Satake compactification $X_{2}^{*}$ by adjoining the 0 -dimensional boundary components in its closure. 


\section{The COHOMOLOGY AND HOMOLOGY}

The material in this section follows [19, §3], [39, §2], and [3, all of which rely on [12]. Recall that $\Gamma=\mathrm{GL}_{N}(\mathcal{O})$. In this section, we introduce a complex $\operatorname{Vor}_{N, D}=\left(V_{*}(\Gamma), d_{*}\right)$ of $\mathbb{Z}[\Gamma]$-modules whose homology is isomorphic to the group cohomology of $\Gamma$ modulo small primes. More precisely, for any positive integer $n$ let $\mathcal{S}_{n}$ be the Serre class of finite abelian groups with orders only divisible by primes less than or equal to $n$ [35]. Then the main result of this section (Theorem 3.7) is that, if $n=n(N, D)$ is larger than all the primes dividing the orders of finite subgroups of $\Gamma$, then modulo $\mathcal{S}_{n}$ the homology of $\operatorname{Vor}_{N, D}$ is isomorphic to the group cohomology of $\Gamma$.

As above let $\Sigma_{n}^{*}=\Sigma_{n}^{*}(\Gamma)$ denote a finite set of representatives, modulo the action of $\Gamma$, of $n$-dimensional cells of $X_{N}^{*}$ that meet $X_{N}$. A cell $\sigma$ is called orientable if every element in $\operatorname{Stab}(\sigma)$ preserves the orientation of $\sigma$. By $\Sigma_{n}=\Sigma_{n}(\Gamma)$ we denote the set of orientable cells in $\Sigma_{n}^{*}(\Gamma)$. Let $\Sigma^{*}=\cup_{n} \Sigma_{n}^{*}$ and let $\Sigma=\cup_{n} \Sigma_{n}$.

3.1. Steinberg homology. The arithmetic group $\Gamma$-like any arithmetic group -is a virtual duality group [6, Theorem 11.4.4]. This means that there is a $\mathbb{Z}[\Gamma]$-module $I$ that plays the role of an "orientation module" for an analogue of Poincaré duality: for all coefficient modules $M$ there is an isomorphism between the cohomology of $\Gamma^{\prime}$ with coefficients in $M$ and the homology of $\Gamma^{\prime}$ with coefficients in $M \otimes I$, for any torsion-free finite index subgroup $\Gamma^{\prime} \subset \Gamma$. For more details see [6, §11]. The module $I$ is called the dualizing module for $\Gamma$. Borel and Serre prove that $I$ is isomorphic to $H^{\nu}(\Gamma, \mathbb{Z}[\Gamma])$, where $\nu=\operatorname{vcd} \Gamma$ is the virtual cohomological dimension of $\Gamma$ (cf. (44); note that for $\Gamma=\mathrm{GL}_{N}(\mathcal{O})$, we have $\nu=N^{2}-N$.

One can show that $I$ is isomorphic to $\mathrm{St}_{\Gamma} \otimes \Omega$, where $\mathrm{St}_{\Gamma}$ is the Steinberg module (the top non-vanishing reduced homology group of the Tits building attached to $\mathrm{GL}_{N} / F$ ), and $\Omega$ is the orientation module (which records whether or not an element changes the orientation of the symmetric space). Since the action of $\mathrm{GL}_{N}(\mathcal{O})$ preserves the orientation of $\mathcal{H}^{N}(\mathbb{C})$, (cf. Remark 2.5), the module $\Omega$ is trivial, and thus $H^{\nu}(\Gamma, \mathbb{Z}[\Gamma])=\mathrm{St}_{\Gamma}$.

For any $\Gamma$-module $M$, the Steinberg homology $\left[12\right.$, p. 279], denoted $H_{*}^{\mathrm{St}}(\Gamma, M)$, is defined as $H_{*}^{\mathrm{St}}(\Gamma, M)=H_{*}\left(\Gamma, \mathrm{St}_{\Gamma} \otimes M\right)$. Let $\hat{H}^{*}$ denote the Farrell cohomology $\hat{H}^{*}(\Gamma, M)$ of $\Gamma$ with coefficients in $M$ (cf. [12, X.3]). There is a long exact sequence

$$
\cdots \rightarrow H_{\nu-i}^{\mathrm{St}} \rightarrow H^{i} \rightarrow \hat{H}^{i} \rightarrow H_{\nu-(i+1)}^{\mathrm{St}} \rightarrow H^{i+1} \rightarrow \hat{H}^{i+1} \rightarrow \cdots
$$

that implies that we can understand the group cohomology by understanding the Steinberg homology and the Farrell cohomology. In particular, if the Farrell cohomology vanishes (which is the case when the torsion primes in $\Gamma$ are invertible in $M$, see [12, IX.9 et seq.]), then the Steinberg homology is exactly the group cohomology $H_{\nu-k}^{\mathrm{St}}(\Gamma, M) \simeq H^{k}(\Gamma, M)$.

We now specialize to the case $M=\mathbb{Z}$ with trivial $\Gamma$-action, and in the remainder of this section omit the coefficients from homology and cohomology groups.

Proposition 3.1. Let $b$ be an upper bound on the torsion primes for $\Gamma=\mathrm{GL}_{N}(\mathcal{O})$. Then, modulo the Serre class $\mathcal{S}_{b}$, we have

$$
H_{\nu-k}^{\mathrm{St}}(\Gamma) \simeq H^{k}(\Gamma) .
$$

3.2. The Voronoi complex. Let $V_{n}(\Gamma)$ denote the free abelian group generated by $\Sigma_{n}(\Gamma)$. Let $d_{n}: V_{n}(\Gamma) \rightarrow V_{n-1}(\Gamma)$ be the map defined in [19, §3.1] (see also Section [6.2), and denote the complex $\left(V_{*}(\Gamma), d_{*}\right)$ by $\operatorname{Vor}_{N, D}$.

Let $\partial X_{N}^{*}$ denote the cells in $X_{N}^{*}$ that do not meet $X$. Then $\partial X_{N}^{*}$ is a $\Gamma$-invariant subcomplex of $X_{N}^{*}$. Let $H_{*}^{\Gamma}\left(X_{N}^{*}, \partial X_{N}^{*}\right)$ denote the relative equivariant homology of the pair $\left(X_{N}^{*}, \partial X_{N}^{*}\right)$ with integral coefficients (cf. [12, VII.7]). 
Proposition 3.2. Let $b$ be an upper bound on the torsion primes for $\Gamma=\mathrm{GL}_{N}(\mathcal{O})$. Modulo the Serre class $\mathcal{S}_{b}$, we have

$$
H_{n}\left(\operatorname{Vor}_{N, D}\right) \simeq H_{n}^{\Gamma}\left(X_{N}^{*}, \partial X_{N}^{*}\right) .
$$

Proof. This result follows from [39, Proposition 2]. The argument is explained in detail for $F=\mathbb{Q}$ in $[19, \S 3.2]$ and can be extended to imaginary quadratic $F$. For the convenience of the reader we recall the argument.

There is a spectral sequence $E_{p q}^{r}$ converging to the equivariant homology groups

$$
H_{p+q}^{\Gamma}\left(X_{N}^{*}, \partial X_{N}^{*}\right)
$$

of the homology pair $\left(X_{N}^{*}, \partial X_{N}^{*}\right)$ such that

$$
E_{p q}^{1}=\bigoplus_{\sigma \in \Sigma_{p}^{*}} H_{q}\left(\operatorname{Stab}(\sigma), \mathbb{Z}_{\sigma}\right) \Rightarrow H_{p+q}^{\Gamma}\left(X_{N}^{*}, \partial X_{N}^{*}\right),
$$

where $\mathbb{Z}_{\sigma}$ is the orientation module for $\sigma$.

When $\sigma$ is not orientable the homology $H_{0}\left(\operatorname{Stab}(\sigma), \mathbb{Z}_{\sigma}\right)$ is killed by 2 . Otherwise, $H_{0}\left(\operatorname{Stab}(\sigma), \mathbb{Z}_{\sigma}\right) \simeq \mathbb{Z}_{\sigma}$. Therefore modulo $\mathcal{S}_{2}$ we have

$$
E_{n, 0}^{1} \simeq \bigoplus_{\sigma \in \Sigma_{n}} \mathbb{Z}_{\sigma}
$$

Furthermore, when $q>0, H_{q}\left(\operatorname{Stab}(\sigma), \mathbb{Z}_{\sigma}\right)$ lies in the Serre class $\mathcal{S}_{b}$, where $b$ is the upper bound on the torsion primes for $\Gamma$. In other words, modulo $\mathcal{S}_{b}$, the $E^{1}$ page of the spectral sequence (4) is concentrated in the bottom row, and there is an identification of the bottom row groups with the groups $V_{n}(\Gamma)$ defined above. Finally, [39, Proposition 2] shows that $d_{n}^{1}=d_{n}$.

Remark 3.3. If we do not work modulo $\mathcal{S}_{b}$, then there are other entries in the spectral sequence to consider before we get $H_{n}^{\Gamma}\left(X_{N}^{*}, \partial X_{N}^{*}\right)$. In particular, the small torsion in $H_{*}\left(\operatorname{Vor}_{N, D}\right)$ may not agree in general with the small torsion in $H_{p+q}^{\Gamma}\left(X_{N}^{*}, \partial X_{N}^{*}\right)$.

3.3. Equivariant relative homology to Steinberg homology. For $V \subset F^{N}$ a proper subspace, let $C(V)$ be the set of matrices $A \in C_{N}^{*}$ such that the kernel of $A$ is $V \otimes_{\mathbb{Q}} \mathbb{R}$, and let $X(V)=\pi(C(V))$. The closure $\overline{C(V)}$ of $C(V)$ in the usual topology induced from $\mathcal{H}^{N}(\mathbb{C})$ consists of matrices whose kernel contains $V$. Let $\overline{X(V)}=\pi(\overline{C(V)})$.

The following lemma shows that $\overline{X(V)}$ is contractible. Soulé proves this for $F=\mathbb{Q}$ in [39, Lemma 1] and [38, Lemma 2], and the same proof with simple modifications applies to our setting as follows.

Lemma 3.4. For any proper subspace $V \subset F^{N}$, the $C W$-complex $\overline{X(V)}$ is contractible.

Proof. Let $A$ be a perfect Hermitian form over $F$. Then $\sigma(A) \cap \overline{X(V)}$ is the perfect cone of the intersection $\operatorname{Min}(A) \cap V^{\perp}$, where $V^{\perp}$ is the orthogonal complement to $V$ in $F^{N}$. It follows that $\overline{X(V)}$ is a sub-CW-complex of $X_{N}^{*}$.

Now we show that $\overline{X(V)}$ is contractible. Since $\overline{X(V)}$ has the same homotopy type as $X(V)$, it is enough to prove that $X(V)$ is contractible. For this it suffices to prove that the CW-topology on $X(V)$ coincides with its usual topology, since $X(V)$ in this topology is clearly contractible (because it is convex). To prove this, we argue that the covering of $X(V)$ by the closed sets of the form $\sigma(A) \cap X(V)$, where $A$ is perfect, is locally finite.

Given any positive definite Hermitian form $A$, let $A^{\perp}$ be its restriction to the real space $V_{\mathbb{R}}^{\perp}=V^{\perp} \otimes_{\mathbb{Q}} \mathbb{R}$. Then $X(V)$ is isomorphic to the symmetric space for the $\operatorname{group} \operatorname{Aut}\left(V_{\mathbb{R}}^{\perp}\right)$ 
via the map $A \mapsto A^{\perp}$. If $\gamma \in \Gamma$ satisfies $\gamma \cdot X(V) \cap X(V) \neq \emptyset$, then in fact $\gamma$ stabilizes $V$. Let $P \subset \Gamma$ be the stabilizer of $V$ and let $\alpha: P \rightarrow \Gamma^{\prime}=\operatorname{Aut}\left(V_{\mathbb{R}}^{\perp} \cap L\right)$ be the projection map. Then the set of cells $\sigma(A) \cap X(V)$, as $A$ ranges over the perfect Hermitian forms, is finite modulo $\Gamma^{\prime}$ : if $[\sigma(A) \cap X(V)] \cap[(\gamma \cdot \sigma(A)) \cap X(V)] \neq \emptyset$, then $\gamma \in P$, and thus $(\gamma \cdot \sigma(A) \cap X(V))=\alpha(\gamma)(\sigma(A) \cap X(V))$.

To conclude the argument one uses Siegel sets; we refer to [7] for their definition and to [4, Ch. II] for their properties in our setting. Given any point $x \in X(V)$, one can find an open set $U \ni x$ and a Siegel set $\mathfrak{S}$ such that $\mathfrak{S} \supset U$. Furthermore, any cell of the form $\sigma(A) \cap X(V)$ is itself contained in another Siegel set $\mathfrak{S}^{\prime}$. Thus if $U$ meets $\gamma(\sigma(A) \cap X(V))$ for $\gamma \in \Gamma^{\prime}$, we must have that $\gamma \cdot \mathfrak{S}^{\prime}$ meets $\mathfrak{S}$. But this is only possible for finitely many $\gamma$ by the standard properties of Siegel sets. Thus the covering of $X(V)$ by the closed sets of the form $\sigma(A) \cap X(V)$ (A perfect) is locally finite, which completes the proof.

Proposition 3.5. For every $n \geq 0$, there are canonical isomorphisms of $\Gamma$-modules

$$
H_{n}\left(X_{N}^{*}, \partial X_{N}^{*}\right) \simeq \begin{cases}\mathrm{St}_{\Gamma} & \text { if } n=N-1, \\ 0 & \text { otherwise. }\end{cases}
$$

Proof. Since $X_{N}^{*}$ is contractible, the long exact sequence for the pair $\left(X_{N}^{*}, \partial X_{N}^{*}\right)$

$$
\cdots \rightarrow H_{n}\left(\partial X_{N}^{*}\right) \rightarrow H_{n}\left(X_{N}^{*}\right) \rightarrow H_{n}\left(X_{N}^{*}, \partial X_{N}^{*}\right) \rightarrow H_{n-1}\left(\partial X_{N}^{*}\right) \rightarrow H_{n-1}\left(X_{N}^{*}\right) \rightarrow \cdots
$$

implies $H_{n}\left(X_{N}^{*}, \partial X_{N}^{*}\right) \simeq \tilde{H}_{n-1}\left(\partial X_{N}^{*}\right)$ for $n \geq 1$, where $\tilde{H}$ denotes reduced homology.

From properties of Hermitian forms it is clear that $\overline{X(V)} \cap \overline{X(W)}=\overline{X(V \cap W)}$ for each pair of proper, non-zero subspaces $V, W \subset F^{N}$. Thus the nerve of the covering $\mathcal{U}$ of $\partial X_{N}^{*}$ by $\overline{X(V)}$ as $V$ ranges over non-zero, proper subspaces of $F^{N}$ is the spherical Tits building $T_{F, N}$. Since each $\overline{X(V)}$ in $\mathcal{U}$ is contractible by Lemma 3.4, the cover $\mathcal{U}$ is a good cover (i.e., nonempty finite intersections are diffeomorphic to $\mathbb{R}^{d}$ for some $d$ ). It follows that the relative homology groups are isomorphic, i.e. $\tilde{H}_{n-1}\left(\partial X_{N}^{*}\right) \simeq \tilde{H}_{n-1}\left(T_{F, N}\right)$. By the Solomon-Tits theorem [37, the latter is isomorphic to $\mathrm{St}_{\Gamma}$ if $n-1=N-2$ and is trivial otherwise.

Proposition 3.6. For all n, we have

$$
H_{n}^{\Gamma}\left(X_{N}^{*}, \partial X_{N}^{*}\right)=H_{n-(N-1)}^{\mathrm{St}}(\Gamma) .
$$

Proof. There is a spectral sequence [39, equation (2)] computing the relative equivariant homology

$$
E_{p q}^{2}=H_{p}\left(\Gamma, H_{q}\left(X_{N}^{*}, \partial X_{N}^{*}\right)\right) \Rightarrow H_{p+q}^{\Gamma}\left(X_{N}^{*}, \partial X_{N}^{*}\right) .
$$

Proposition 3.5 implies the $E^{2}$ page of the spectral sequence is concentrated in the $q=N-1$ column. Then

$$
H_{p+(N-1)}^{\Gamma}\left(X_{N}^{*}, \partial X_{N}^{*}\right) \simeq H_{p}\left(\Gamma, H_{q}\left(X_{N}^{*}, \partial X_{n}^{*}\right)\right)=H_{p}\left(\Gamma, \mathrm{St}_{\Gamma}\right)=H_{p}^{\mathrm{St}}(\Gamma),
$$

and the result follows.

Theorem 3.7. Let $b$ be an upper bound on the torsion primes for $\mathrm{GL}_{N}(\mathcal{O})$. Modulo the Serre class $\mathcal{S}_{b}$, one has

$$
H_{n}\left(\operatorname{Vor}_{N, D}\right) \simeq H^{N^{2}-1-n}\left(\mathrm{GL}_{N}(\mathcal{O})\right) .
$$

Proof. Let $\Gamma=\mathrm{GL}_{N}(\mathcal{O})$. Modulo $\mathcal{S}_{b}$, Propositions 3.2, 3.6, and 3.1 imply

$$
H_{n}\left(\operatorname{Vor}_{N, D}\right) \simeq H_{n}^{\Gamma}\left(X_{N}^{*}, \partial X_{N}^{*}\right) \simeq H_{n-(N-1)}^{\mathrm{St}}(\Gamma) \simeq H^{N^{2}-1-n}(\Gamma) .
$$


3.4. Torsion elements in $\Gamma$. To finish this section we discuss the possible torsion that can arise in the stabilizer subgroups of cells in our complexes. This allows us to make the bound in Theorem 3.7 effective.

Lemma 3.8. Let $p$ be an odd prime, and let $F / \mathbb{Q}$ be a quadratic field. Let $\Phi_{p}=x^{p-1}+$ $x^{p-2}+\cdots+x+1$ be the $p^{\text {th }}$ cyclotomic polynomial. Then $\Phi_{p}$ factors over $F$ as a product of irreducible polynomials of degree $(p-1) / 2$ if $F=\mathbb{Q}\left(\sqrt{p^{*}}\right)$, where $p^{*}=(-1)^{(p-1) / 2} p$, and is irreducible otherwise.

Proof. Let $\zeta_{p}$ denote a primitive $p^{\text {th }}$ root of unity. Consider the diagram of Galois extensions below:

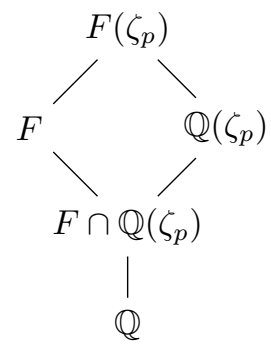

If $F=\mathbb{Q}\left(\sqrt{p^{*}}\right)$, then $F$ is the unique quadratic subfield of $\mathbb{Q}\left(\zeta_{p}\right)$. It follows that $\Phi_{p}$ factors over $F$ as a product of irreducible polynomials of degree $(p-1) / 2$. If $F \neq \mathbb{Q}\left(\sqrt{p^{*}}\right)$, then $F \cap \mathbb{Q}\left(\zeta_{p}\right)=\mathbb{Q}$. It follows that $\left[F\left(\zeta_{p}\right): \mathbb{Q}\left(\zeta_{p}\right)\right]=[F: \mathbb{Q}]=2$ and so $\left[F\left(\zeta_{p}\right): F\right]=p-1$. Thus $\Phi_{p}$ is irreducible over $F$ if $F \neq \mathbb{Q}\left(\sqrt{p^{*}}\right)$.

Lemma 3.9. Let $p$ be an odd prime, and let $F / \mathbb{Q}$ be a imaginary quadratic field. If $g \in$ $\mathrm{GL}_{N}(F)$ has order $p$, then

$$
p \leq \begin{cases}N+1 & \text { if } p \equiv 1 \bmod 4 \\ 2 N+1 & \text { otherwise. }\end{cases}
$$

Proof. If $p \equiv 1 \bmod 4$, then $p^{*}>0$. In particular, $F \neq \mathbb{Q}\left(\sqrt{p^{*}}\right)$ and so by Lemma 3.8, $\Phi_{p}$ is irreducible over $F$. Then the minimal polynomial of $g$ is $\Phi_{p}$. By the Cayley-Hamilton Theorem, $\Phi_{p}$ divides the characteristic polynomial of $g$. Therefore $p-1 \leq N$. Similarly, if $p \not \equiv 1 \bmod 4$, then $(p-1) / 2 \leq N$.

Lemmas 3.8 and 3.9 immediately imply the following:

Proposition 3.10. If $g \in \mathrm{GL}_{3}\left(\mathcal{O}_{F}\right)$ has prime order $q$, then $q \in\{2,3,7\}$ for $F=\mathbb{Q}(\sqrt{-7})$ and $q \in\{2,3\}$ otherwise. If $g \in \mathrm{GL}_{4}\left(\mathcal{O}_{F}\right)$ has prime order $q$, then $q \in\{2,3,5,7\}$ for $F=\mathbb{Q}(\sqrt{-7})$ and $q \in\{2,3,5\}$ otherwise.

In Tables 1412 we give the factorizations of the orders of the stabilizers of the cells in $\Sigma^{*}$.

\section{A mass formula for the Voronoi COMPleX}

The computation of the cell complex is a relatively difficult task and there are many ways that small mistakes in the computation could cause the final answer to be incorrect. Hence it is very important to have checks that allow us to give strong evidence for the correctness of our computations. One is that the complexes we construct actually are chain complexes, namely that their differentials square to zero. Another is the mass formula, stated in Theorem 4.6. According to this formula, the alternating sum over the cells of 
$\Sigma^{*}$ of the inverse orders of the stabilizer subgroups must vanish. A good reference for this section is [12, Ch. IX, $\S \S 6-7]$, and we follow it closely. The main theorem underlying this computation is due to Harder [21].

4.1. Euler characteristics. We begin by recalling some definitions. The cohomological dimension $\operatorname{cd} \Gamma$ of a group $\Gamma$ is the largest $n \in \mathbb{Z} \cup\{\infty\}$ such that there exists a $\mathbb{Z} \Gamma$-module $M$ with $H^{n}(\Gamma ; M) \neq 0$. The virtual cohomological dimension $\operatorname{vcd} \Gamma$ of $\Gamma$ is defined to be the cohomological dimension of any torsion-free finite index subgroup of $\Gamma$ (one can show that this is well-defined). We recall that $\Gamma$ is said to be of finite homological type if (i) $\operatorname{vcd} \Gamma<\infty$ and (ii) for every $\mathbb{Z} \Gamma$-module $M$ that is finitely generated as an abelian group, the homology group $H_{i}(\Gamma ; M)$ is finitely generated for all $i$.

Definition 4.1. Let $\Gamma$ be a torsion-free group of finite homological type, and let $H_{*}(\Gamma)$ denote the homology of $\Gamma$ with (trivial) $\mathbb{Z}$-coefficients. The Euler characteristic $\chi(\Gamma)$ is

$$
\chi(\Gamma)=\sum_{i}(-1)^{i} \operatorname{rank}_{\mathbb{Z}}\left(H_{i}(\Gamma)\right) .
$$

Proposition 4.2. [12, Theorem 6.3] If $\Gamma$ is torsion-free and of finite homological type and $\Gamma^{\prime} \subseteq \Gamma$ is a subgroup of finite index, then

$$
\chi\left(\Gamma^{\prime}\right)=\left[\Gamma: \Gamma^{\prime}\right] \cdot \chi(\Gamma) .
$$

One can use Proposition 4.2 to extend the notion of Euler characteristic to groups with torsion. Namely, if $\Gamma$ is an arbitrary group of finite homological type with a torsion-free subgroup $\Gamma^{\prime}$ of finite index, one sets

$$
\chi(\Gamma)=\frac{\chi\left(\Gamma^{\prime}\right)}{\left[\Gamma: \Gamma^{\prime}\right]} .
$$

By Proposition 4.2, this is independent of the choice of $\Gamma^{\prime}$.

Proposition 4.3. If $\Gamma$ is a finite group, then $\chi(\Gamma)=1 /|\Gamma|$.

Proof. Take $\Gamma^{\prime}$ to be the trivial subgroup in (5).

Theorem 4.4. [21] Let $F$ be a number field with ring of integers $\mathcal{O}$, and let $\zeta_{F}(s)$ be the Dedekind zeta function of $F$. Then

$$
\chi\left(\mathrm{SL}_{N}(\mathcal{O})\right)=\prod_{k=2}^{N} \zeta_{F}(1-k) .
$$

In particular, since for imaginary quadratic $F$ the Dedekind zeta function $\zeta_{F}(m)$ vanishes for all $m \in \mathbb{Z}<0$, we have that $\chi\left(\mathrm{SL}_{N}(\mathcal{O})\right)=0$ when $N \geq 2$.

Corollary 4.5. Let $F$ be an imaginary quadratic field with ring of integers $\mathcal{O}$. Then $\chi\left(\mathrm{GL}_{N}(\mathcal{O})\right)=0$ when $N \geq 2$.

Proof. This follows immediately from Theorem 4.4 and the definition of the Euler characteristic since $\mathrm{SL}_{N}(\mathcal{O})$ is of finite index in $\mathrm{GL}_{N}(\mathcal{O})$. 
4.2. The mass formula. Now we turn to a different concept, the equivariant Euler characteristic $\chi_{\Gamma}(X)$. Let $X$ be any cell complex with $\Gamma$ action such that (i) $X$ has finitely many cells mod $\Gamma$, and (ii) for each $\sigma \in X$, the stabilizer subgroup $\operatorname{Stab}_{\Gamma}(\sigma)$ is finite. One defines

$$
\chi_{\Gamma}(X)=\sum_{\sigma \in S}(-1)^{\operatorname{dim} \sigma} \chi\left(\operatorname{Stab}_{\Gamma}(\sigma)\right),
$$

where $S$ is a set of representatives of cells of $X \bmod \Gamma$. One knows that if $\Gamma$ has a torsion-free subgroup of finite index, and the cell complex $X$ is contractible, then in fact $\chi_{\Gamma}(X)=\chi(\Gamma)$ [12, Proposition $\left.7.3\left(\mathrm{e}^{\prime}\right)\right]$.

The well-rounded retract $W$ defined in $\$ 2.2$ is a proper, contractible $\Gamma$-complex and so its equivariant Euler characteristic is defined. We compute its equivariant Euler characteristic, phrased in terms of cells in $\Sigma^{*}$ using Corollary 2.9, to get a mass formula.

Theorem 4.6 (Mass Formula). We have

$$
\sum_{\sigma \in \Sigma_{k}^{*}}(-1)^{k} \frac{1}{\left|\operatorname{Stab}_{\Gamma}(\sigma)\right|}=0 .
$$

Proof. Let $\Gamma=\mathrm{GL}_{N}(\mathcal{O})$. By Proposition 4.3 we have $\chi\left(\operatorname{Stab}_{\Gamma}(\sigma)\right)=1 /\left|\operatorname{Stab}_{\Gamma}(\sigma)\right|$. Furthermore, by Theorem 2.7 and Corollary 2.9] we can identify cells of $W \bmod \Gamma$ with cells in $\Sigma^{*}$. Thus (6) represents the equivariant Euler characteristic $\chi_{\Gamma}(W)$. Since $W$ is contractible [1,2] and $\Gamma$ has torsion-free subgroups of finite index (any sufficiently small congruence subgroup is torsion-free), the remark at the end of $\$ 4.1$ implies $\chi_{\Gamma}(W)=\chi(\Gamma)$. Finally, by Corollary 4.5] we have $\chi(\Gamma)=0$, which completes the proof.

Using the stabilizer information in Tables 112 one can easily verify that $\chi\left(\mathrm{GL}_{n}(\mathcal{O})\right)=0$ for each of our examples. The most spectacular example is $\mathrm{GL}_{4}\left(\mathcal{O}_{-4}\right)$. If we add together the terms $1 /\left|\operatorname{Stab}_{\Gamma}(\sigma)\right|$ for cells $\sigma$ of the same dimension to a single term for $\operatorname{GL}_{4}\left(\mathcal{O}_{-4}\right)$, we find (ordering the terms by increasing dimension in $\Sigma^{*}$ )

$$
\begin{aligned}
-\frac{11}{3072}+\frac{127}{960}-\frac{4187}{2304}+\frac{28375}{2304}-\frac{868465}{18432}+\frac{126127}{1152}-\frac{81945}{512} \\
+\frac{340955}{2304}-\frac{48655}{576}+\frac{16075}{576}-\frac{21337}{4608}+\frac{101}{384}-\frac{17}{92160}=0 .
\end{aligned}
$$

The other groups have been checked similarly.

\section{Explicit homology Classes}

By Theorem 3.7 we have $H_{N^{2}-1}\left(\operatorname{Vor}_{N, D} \otimes \mathbb{Q}\right) \simeq H^{0}\left(\mathrm{GL}_{N}(\mathcal{O}), \mathbb{Q}\right)$, which in turn is isomorphic to $\mathbb{Q}$. This suggests that there should be a canonical generator for this homology group, a fact already explored in [19, Section 5]. An obvious choice is the analogue of the chain presented there, namely

$$
\xi:=\xi_{N, D}:=\sum_{\sigma} \frac{1}{|\operatorname{Stab}(\sigma)|}[\sigma],
$$

where $\sigma$ runs through the cells in $\Sigma_{N^{2}-1}\left(\mathrm{GL}_{N}(\mathcal{O})\right)$. In this section we verify that this is true for our examples. We should point out that all the cells in $\Sigma_{N^{2}-1}\left(\mathrm{GL}_{N}(\mathcal{O})\right)$ are orientable. The reason is that the group $\mathrm{GL}_{n}(\mathbb{C})$ is connected and so the determinant of its action on $\mathcal{H}^{N}(\mathbb{C})$ is positive. Since the stabilizers are included in $\mathrm{GL}_{N}(\mathcal{O})$ and the faces in $\Sigma_{N^{2}-1}\left(\mathrm{GL}_{N}(\mathcal{O})\right)$ are full-dimensional, the orientation has to be preserved. 
Theorem 5.1. When $N=3$ and $D \geq-24$ or $N=4$ and $D=-3,-4$, the chain $\xi$ is a cycle and thus generates $H_{N^{2}-1}\left(\operatorname{Vor}_{N, D} \otimes \mathbb{Q}\right)$.

Proof. The proof is an explicit computation with differential matrix $A$ representing the map $V_{N^{2}-1}(\Gamma) \rightarrow V_{N^{2}-2}(\Gamma)$ (cf. 33.2 ). Note that the signs of the entries of $A$ depend on a choice of orientation for each of the cells in $\Sigma_{N^{2}-1}$ and $\Sigma_{N^{2}-2}$. In each non-zero row of $A$ there are exactly two non-zero entries. Each non-zero entry $A_{i, j}$ has absolute value $\left|\operatorname{Stab}\left(\sigma_{j}\right)\right| /\left|\operatorname{Stab}\left(\tau_{i}\right)\right|$, where $\sigma_{j} \in \Sigma_{N^{2}-1}\left(\mathrm{GL}_{N}(\mathcal{O})\right)$ and $\tau_{i} \in \Sigma_{N^{2}-2}\left(\mathrm{GL}_{N}(\mathcal{O})\right)$. One then checks that there is a choice of orientations such that the non-zero entries in a given row have opposite signs.

For example, consider $N=4$ and $D=-4$. The relevant differential matrix is

$$
d_{15}=\left[\begin{array}{cc}
0 & 0 \\
1920 & -256
\end{array}\right]
$$

with kernel generated by $(2,15)=92160(1 / 46080,1 / 6144)$. The orders of the two stabilizer groups for the cells in $\Sigma_{15}\left(\mathrm{GL}_{N}\left(\mathcal{O}_{-4}\right)\right)$ are 46080 and 6144 , respectively, and thus $\xi_{4,-4}$ is a cycle.

Remark 5.2. It seems likely that Theorem 5.1 holds for all $\mathrm{GL}_{N}\left(\mathcal{O}_{D}\right)$, although we do not presently have a proof.

\section{Algorithms}

In this section we give more details about the specific algorithms we used in this project. There are several stages needed to compute the Voronoi homology:

(1) Determination of the perfect forms.

(2) Computation of the Voronoi complex and its differentials.

(3) Computation of the homology.

We discuss each one of these below in turn.

We remark that all our computations were performed with two different implementations, one in MAGMA 9 and one in GAP 43 . In addition the determination of the perfect forms was also done using a C++ 42 implementation currently under development that we plan to use as our main codebase in the future. The first two implementations produced identical results, and the third implementation successfully recovered the list of perfect forms. Complete source code for the GAP implementation, including data for all the Voronoi complexes, can be found at [14. Another version of the source code, which does only the cohomology of perfect forms, can be found at 17 .

6.1. Determination of the perfect forms. Recall that $\mathcal{H}^{N}(\mathbb{C})$ is the vector space of Hermitian forms in $N$ variables, and that each field $F$ endows this space with a rational structure. We begin by describing a model for $\mathcal{H}^{N}(\mathbb{C})$ that allows us to perform all computations with exact arithmetic. To do this we first represent the space $\mathcal{H}^{N}(\mathbb{C})$ as a vector space of symmetric $2 N \times 2 N$ real matrices.

Let $\mathcal{S}^{N}$ the space of $N \times N$ real symmetric matrices and $\mathcal{A} S^{N}$ the space of $N \times N$ real antisymmetric matrices. Considering the action of complex conjugation, we write a matrix $A \in \mathcal{H}^{N}(\mathbb{C})$ as $A=U+V$ with $U \in \mathcal{S}^{N}$ and $V \in \mathcal{A} S^{N}$. Then for any $x, y \in \mathbb{Z}^{N}$, we have

$$
\begin{aligned}
A[x+\omega y] & =(x+\omega y)^{*} A(x+\omega y) \\
& =x^{T} U x+P y^{t} U y+x^{t} S U y+x^{T}\left(\omega-\omega^{*}\right) V y,
\end{aligned}
$$


where $P=\alpha^{*} \alpha$ and $S=\alpha+\alpha^{*}$, and $\omega \in F$ is defined at the beginning of Section 2 Putting $W=\left(\omega-\omega^{*}\right) V / 2$, we can define the space

$$
\mathcal{S} P(\mathbb{Z}[\omega]):=\left\{\left(\begin{array}{cc}
U & (S / 2) U+W \\
(S / 2) U+W & P U
\end{array}\right) \in \mathcal{S}^{2 N} \text { for } U \in \mathcal{S}^{N} \text { and } W \in \mathcal{A} S^{N}\right\} .
$$

The space $\mathcal{S} P(\mathbb{Z}[\omega])$ has a natural basis of $\mathbb{Q}$-rational matrices, and using this basis, all our computations can be carried out over the integers. With this model for $\mathcal{H}^{N}(\mathbb{C})$, the positive definite elements of $\mathcal{S} P(\mathbb{Z}[\omega])$ correspond to the positive definite Hermitian forms, and the group $\Gamma=\mathrm{GL}_{N}(\mathcal{O})$ is a subgroup of $\mathrm{GL}_{2 N}(\mathbb{Z})$. We remark that this "restriction of scalars" construction can be carried out for general number fields $F$, but that one obtains rational spaces only when $F$ is totally real or CM.

Thus we are reduced to performing computations in a space of real symmetric matrices and its subcone of positive definite matrices. By now there is an extensive literature giving tools to work with such spaces. For instance, specific algorithms have been developed in [32] for enumerating the set of perfect forms when it is finite; we will only briefly restate them here and refer to 32 for detailed definitions and algorithms. Another good source is [36].

As in Definition 2.1, let $\operatorname{Min}(A)$ be the set of minimal vectors of a positive definite form $A \in \mathcal{S}^{N}$. The key algorithmic functions available for a given space $\mathcal{S} P$ of symmetric matrices are as follows:

(1) Enlarging sets of miminal vectors. Let $A$ be a positive definite quadratic form and let $v \in \operatorname{Min}(A)$ be a minimal vector. Suppose $B \neq 0$ is a quadratic form with $B[v]=0$. Then we can find $\lambda>0$ such that $\operatorname{Min}(A)$ is properly contained in $\operatorname{Min}(A+\lambda B)$. [32, Algorithm 2].

(2) Finding perfect forms. Starting with a positive definite form $A$ and its set of minimal vectors $\operatorname{Min}(A)$, we can iterate the enlarging algorithm [32, Algorithm 2] until we obtain a perfect form [32, Page 371].

(3) Finding the cone of a perfect form. Given one perfect form $A$ we can compute the cone $\sigma(A)$ it determines and then the facets of $\sigma(A)$ using the Recursive Adjacency Decomposition Method [36, Section 3.2], 31.

(4) Finding neighbors of perfect forms. Suppose $\tau$ is a facet of a perfect cone $\sigma(A)$. Let $\operatorname{Min}(\tau) \subset \operatorname{Min}(A)$ be the set of corresponding shortest vectors. Up to a positive multiple there is a unique matrix $B \in \mathcal{S} P$ such that (i) $B[v]=0$ for $v \in \operatorname{Min}(\tau)$ and (ii) $B[v]>0$ for $v \in \operatorname{Min}(A) \backslash \operatorname{Min}(\tau)$. In other words, $B$ determines a linear form that cuts $\tau$ out from $\sigma(A)$ and is positive on $\sigma(A) \backslash \tau$. If $B$ is not positive definite then there is a perfect form $A^{\prime}$ such that $\sigma\left(A^{\prime}\right) \cap \sigma(A)=\tau$; we say $A^{\prime}$ is a neighbor of $A$. Any neighboring form of $A$ can be easily determined using the enlarging algorithm [32, Algorithm 2]. On the other hand, if $B$ is positive definite, then one can show that there is no perfect form neighboring $A$ along $\tau$. In this case the facet $\tau$ is called a dead end [32. In all our cases considered here, there were no dead ends.

(5) Equivalence and automorphisms. In order to compute with perfect forms, it is indispensable to be able to determine when two forms lie in the same $\Gamma$-orbit and to determine a form's stabilizer in $\Gamma$. In the classical setting of positive definite forms over $\mathbb{Q}$, the best tools are the algorithms ISOM / AUTO due to Plesken-Souvignier [27. Applying these algorithms in our setting using the model $\mathcal{S} P(\mathbb{Z}[\omega])$ produces stabilizers that are too large and false equivalences, since in our case we only want the matrices $P \in \mathrm{GL}_{2 N}(\mathbb{Z})$ that belong to the embedding of $\Gamma$ into $\mathrm{GL}_{2 N}(\mathbb{Z})$. Adaptations of ISOM / AUTO allowing this are described in in [11, Example 5]. 
By combining the preceding algorithms, one obtains the generalization of Voronoi's classical algorithm that allows one to find all the perfect forms in the cone of positive definite Hermitian forms up to $\Gamma$-equivalence. Details are given in $[31,32,36$.

6.2. Computing the Voronoi complex and the differentials. After the steps in Section 6.1 are finished, we have obtained a list of representatives of the perfect forms and their stabilizer groups. Once one has this list of perfect forms, one essentially has the set of top-dimensional cells of $\Sigma^{*}(\Gamma)$.

One then needs to determine the full polyhedral structure of all these cells. This is done in an obvious way using well known algorithms in polyhedral geometry: one finds all the facets of a given cell, determines their facets, and so on. The subtlety in the process lies in testing equivalence of cells. Ultimately one is only interested in those faces that do not lie at infinity (i.e. those that meet $\left.X_{N}\right)$. Given two cells $\tau, \tau^{\prime} \in \Sigma_{k}^{*}(\Gamma)$, one first computes two barycenter matrices $\operatorname{Cent}(\tau)$, Cent $\left(\tau^{\prime}\right)$ by taking the sums of their corresponding extreme rays. Since the cells $\tau, \tau^{\prime}$ meet $X_{N}$, the matrices $\operatorname{Cent}(\tau)$ and $\operatorname{Cent}\left(\tau^{\prime}\right)$ are positive definite. Thus we can check whether or not they are equivalent using the same methods involved for enumerating perfect cones.

After carrying this out, the result is the full set $\Sigma^{*}(\Gamma)$, along with information about inclusion relations between cells. This information is exactly what one needs to compute the differential in the Voronoi complex, although there are subtleties in determining signs. Recall from Section 3 that $\Sigma_{k}(\Gamma) \subset \Sigma_{k}^{*}(\Gamma)$ is the subset of all $k$-cells whose stabilizers are orientation-preserving. The groups in the Voronoi complex are then the free abelian groups $V_{k}(\Gamma)$ generated by the elements of $\Sigma_{k}(\Gamma)$, and we must determine the differential $d_{k}: V_{k}(\Gamma) \rightarrow V_{k-1}(\Gamma)$. This is done as follows.

For each closed cell $\sigma$ in $X_{N}^{*}$, we first fix an orientation of $\sigma$, i.e. an orientation of the real vector space $\mathbb{R}(\sigma)$ of symmetric matrices spanned by the forms $q(v)$ with $v \in \operatorname{Min}(\sigma)$. Let $\sigma \in \Sigma_{k}$ and let $\tau^{\prime}$ be a face of $\sigma$ that is equivalent under $\Gamma$ to an element in $\Sigma_{k-1}$ (i.e. $\tau^{\prime}$ neither lies on the boundary nor has elements in its stabilizer reversing the orientation). Given a positive basis $B^{\prime}$ of $\mathbb{R}\left(\tau^{\prime}\right)$ we get a basis $B$ of $\mathbb{R}(\sigma) \supset \mathbb{R}\left(\tau^{\prime}\right)$ by appending to $B^{\prime}$ the vector $\operatorname{Cent}(\sigma)$. We let $\varepsilon\left(\tau^{\prime}, \sigma\right)= \pm 1$ be the sign of the orientation of $B$ in the oriented vector space $\mathbb{R}(\sigma)$.

Next, let $\tau \in \Sigma_{k-1}$ be the (unique) cell equivalent to $\tau^{\prime}$ and let $\gamma \in \Gamma$ be such that $\tau^{\prime}=\tau \cdot \gamma$. We define $\eta\left(\tau, \tau^{\prime}\right)=1$ (resp. $\eta\left(\tau, \tau^{\prime}\right)=-1$ ) when $\gamma$ is compatible (resp. incompatible) with the chosen orientations of $\mathbb{R}(\tau)$ and $\mathbb{R}\left(\tau^{\prime}\right)$.

Finally we define, as in [19, the differential by

$$
d_{k}(\sigma)=\sum_{\tau \in \Sigma_{n-1}} \sum_{\tau^{\prime}} \eta\left(\tau, \tau^{\prime}\right) \varepsilon\left(\tau^{\prime}, \sigma\right) \tau .
$$

6.3. Computing the homology. After one has the differentials, computing the cohomology is a standard problem in exact linear algebra. Any cohomology group $H$ is obtained in the form $H=\operatorname{Ker}(A) / \operatorname{Im}(B)$. However, it is important to note that one does not need actually to find the kernel explicitly and compute the quotient. For the rational part of the cohomology, one simply needs the ranks of the matrices $A$ and $B$. For the torsion of $H$, one simply needs the invariant factors of the Smith normal form of $B$ (see the discussion after Theorem 7.3 below). For both computations, the linbox library 23 for exact linear algebra is adequate and is able to take advantage of the natural sparsity of the matrices of the differentials. 


\section{Results AND taBles}

We conclude by presenting the results of our computations. First we summarize the most interesting numerical results from the tables, namely those involving the homology computations for $\mathrm{GL}_{*}\left(\mathcal{O}_{D}\right)$.

Theorem 7.1. Let $0>D \geq-24$ be a fundamental discriminant.

(1) The Voronoi homology $H_{n}\left(\operatorname{Vor}_{3, D}\right)$ can have non-trivial rank only for $n=2,4,5$, and 8.

(2) In each case for $n=8$ we have $H_{8}\left(\operatorname{Vor}_{3, D}\right) \cong \mathbb{Z}$.

(3) In homology degree 2, 4 and 5, we have the following table of ranks (empty slots being zero)

\begin{tabular}{|c|rrrrrrrrrr|}
\hline$D$ & -3 & -4 & -7 & -8 & -11 & -15 & -19 & -20 & -23 & -24 \\
\hline \# $\{$ perf. forms $\}$ & 2 & 1 & 2 & 2 & 12 & 90 & 157 & 212 & 870 & 596 \\
\hline $\operatorname{rank} H_{2}$ & & & & & & & & 1 & 4 & 1 \\
rank $H_{4}$ & 1 & 1 & 1 & 1 & 1 & 3 & 2 & 4 & 5 & 5 \\
rank $H_{5}$ & 1 & 1 & 2 & 2 & 2 & 5 & 3 & 6 & 1 & 7 \\
\hline
\end{tabular}

(4) The p-Sylow subgroups for the primes occurring in (the orders of) the respective homology groups are given in the following table, where we use the shorthand $n(G)$ to denote that the p-Sylow subgroup in $H_{n}\left(\operatorname{Vor}_{3, D}\right)$ is equal to $G$. We also use the "topologist's convention" that $\mathbb{Z}_{n}$ denotes the finite cyclic group $\mathbb{Z} / n \mathbb{Z}$.

\begin{tabular}{|c|cccccccc|}
\hline$D$ & -3 & -4 & -7 & -8 & -11 & -15 & -19 & -20 \\
\hline Syl $_{2}$ & & $5\left(\mathbb{Z}_{2}\right)$ & & $4\left(\mathbb{Z}_{2}\right), 5\left(\mathbb{Z}_{2}\right)$ & $5\left(\mathbb{Z}_{4}\right)$ & $4\left(\mathbb{Z}_{4}\right)$ & $5\left(\mathbb{Z}_{2} \times \mathbb{Z}_{8}\right)$ & $3\left(\mathbb{Z}_{2}\right), 4\left(\mathbb{Z}_{2}^{4}\right), 7\left(\mathbb{Z}_{2}^{2} \times \mathbb{Z}_{4}\right)$ \\
Syl $_{3}$ & $7\left(\mathbb{Z}_{9}\right)$ & & $7\left(\mathbb{Z}_{3}\right)$ & & $7\left(\mathbb{Z}_{3}^{2}\right)$ & $7\left(\mathbb{Z}_{3}^{5}\right)$ & $7\left(\mathbb{Z}_{3}^{4}\right)$ & $5\left(\mathbb{Z}_{3}\right), 7\left(\mathbb{Z}_{3}^{5}\right)$ \\
Syl $_{5}$ & & & & & & $7\left(\mathbb{Z}_{5}\right)$ & & \\
Syl $_{7}$ & & & $5\left(\mathbb{Z}_{7}\right)$ & & & & & $7\left(\mathbb{Z}_{7}\right)$ \\
\hline
\end{tabular}

\begin{tabular}{|c|cc|}
\hline$D$ & -23 & -24 \\
\hline $\mathrm{Syl}_{2}$ & $3\left(\mathbb{Z}_{2}\right), 4\left(\mathbb{Z}_{2}^{13}\right), 5\left(\mathbb{Z}_{2}^{5}\right), 7\left(\mathbb{Z}_{2}^{3} \times \mathbb{Z}_{4}^{2}\right)$ & $3\left(\mathbb{Z}_{2}^{7}\right), 4\left(\mathbb{Z}_{2}^{5}\right), 5\left(\mathbb{Z}_{2}^{8} \times \mathbb{Z}_{4}^{2}\right), 6\left(\mathbb{Z}_{2}\right), 7\left(\mathbb{Z}_{2}^{4} \times \mathbb{Z}_{4}^{2}\right)$ \\
Syl $_{3}$ & $4\left(\mathbb{Z}_{3}^{3}\right), 7\left(\mathbb{Z}_{3}^{7}\right)$ & $7\left(\mathbb{Z}_{3}^{3}\right)$ \\
\hline
\end{tabular}

Theorem 7.2. Let $V_{4}$ be either the Voronoi complex for $\mathrm{GL}_{4}\left(\mathcal{O}_{-3}\right)$ or $\mathrm{GL}_{4}\left(\mathcal{O}_{-4}\right)$. Then $H_{n}\left(V_{4}\right)$ has non-trivial rank only for $n=6,7,9,10,12$, and 15 . More precisely,

(1) For $n=3 k$ we have $\operatorname{rank} H_{3 k}\left(V_{4}\right)= \begin{cases}1 & \text { if } k>1, \\ 0 & \text { if } k=1 .\end{cases}$

(2) For $n=7$ we have $\operatorname{rank} H_{n}\left(V_{4}\right)=2$.

(3) For $n=10$ we have rank $H_{n}\left(V_{4}\right)=1$.

Moreover, the only torsion primes appearing are 2, 3 and 5 .

Invoking Theorem 3.7 we also get information on the cohomology of $\mathrm{GL}_{4}\left(\mathcal{O}_{D}\right)$ :

Theorem 7.3. Let $D=-3$ or -4 . Then $H^{n}\left(\mathrm{GL}_{4}\left(\mathcal{O}_{D}\right)\right)$ has non-trivial rank only for $n=0,3,5,6,8$, and 9 . More precisely,

(1) For $n=3 k$ we have $\operatorname{rank} H^{n}\left(\mathrm{GL}_{4}\left(\mathcal{O}_{D}\right)\right)=1$ if $0 \leq k \leq 3$. 
(2) For $n=5$ we have rank $H^{n}\left(\mathrm{GL}_{4}\left(\mathcal{O}_{D}\right)\right)=1$.

(3) For $n=8$ we have $\operatorname{rank} H^{n}\left(\mathrm{GL}_{4}\left(\mathcal{O}_{D}\right)\right)=2$.

The remainder of the text is devoted to presenting details about the Voronoi complexes and full information about their homology. The notation is as follows:

- The first three columns concern the cell decomposition of $X_{N}^{*} \bmod \Gamma$ :

$-n$ is dimension of the cells in the (partially) compactified symmetric space $X_{N}^{*}$.

$-\left|\Sigma_{n}^{*}\right|$ is the number of $\Gamma$-orbits in the cells that meet $X_{N} \subset X_{N}^{*}$.

- $\mid$ Stab $\mid$ gives the sizes of the stabilizer subgroups in factored form. The notation $A(k)$ means that, of the $\left|\Sigma_{n}^{*}\right|$ cells of dimension $n, k$ of them have a stabilizer subgroup of order $A$.

- The next four columns concern the differentials $d_{n}$ of the Voronoi complex $\operatorname{Vor}_{N, D}$ :

$-\left|\Sigma_{n}\right|$ is the number of orientable $\Gamma$-orbits in $\Sigma_{n}^{*}$.

- nnz is the number of non-zero entries in the differential $d_{n}: V_{n}(\Gamma) \rightarrow V_{n-1}(\Gamma)$.

- rank is the rank of $d_{n}$.

- elem. div. gives the elementary divisors of $d_{n}$. As in the stabilizer column, the notation $d(k)$ means that the elementary divisor $d$ occurs with multiplicity $k$. If the rank of $d_{n}$ is zero then this column is left empty.

- Finally, the last column gives the homology of the Voronoi complex. One can easily check that $H_{n} \simeq \mathbb{Z}^{r} \oplus \bigoplus(\mathbb{Z} / d \mathbb{Z})^{k}$, where $r=\left|\Sigma_{n}\right|-\operatorname{rank}\left(d_{n}\right)-\operatorname{rank}\left(d_{n+1}\right)$ and the sum is taken over the elementary divisors $d(k)$ from row $n+1$. As above, to save space we denote the finite cyclic group $\mathbb{Z} / d \mathbb{Z}$ by $\mathbb{Z}_{d}$. By Theorem 3.7 we have $H_{n}\left(\operatorname{Vor}_{N, D}\right) \simeq H^{N^{2}-1-n}\left(\mathrm{GL}_{N}(\mathcal{O})\right)$ modulo the torsion primes in $\operatorname{GL}_{N}(\mathcal{O})$. These primes are visible in the third column of each table.

TABLE 1. The cell complex, differentials, and homology for $\mathrm{GL}_{3}\left(\mathcal{O}_{-3}\right)$.

\begin{tabular}{|c||c|l||c|c|c|c|r|}
\hline$n$ & $\left|\Sigma_{n}^{*}\right|$ & $\mid$ Stab $\mid$ & $\left|\Sigma_{n}\right|$ & nnz & rank & elem. div. & $H_{n}$ \\
\hline 2 & 1 & $2^{4} 3^{4}(1)$ & 0 & 0 & 0 & & 0 \\
\hline 3 & 2 & $2^{4} 3^{2}(1), 2^{3} 3^{3}(1)$ & 0 & 0 & 0 & & 0 \\
\hline 4 & 3 & $2^{2} 3^{2}(1), 2^{4} 3^{3}(1), 2^{4} 3^{1}(1)$ & 1 & 0 & 0 & & $\mathbb{Z}$ \\
\hline 5 & 4 & $2^{4} 3^{2}(1), 2^{2} 3^{2}(1), 2^{1} 3^{2}(1)$, & 2 & 0 & 0 & & $\mathbb{Z}$ \\
& & $2^{2} 3^{3}(1)$ & 1 & 2 & 1 & $1(1)$ & 0 \\
\hline 6 & 3 & $2^{2} 3^{2}(1), 2^{1} 3^{2}(1), 2^{2} 3^{3}(1)$ & 1 & 0 & 0 & & $\mathbb{Z}_{9}$ \\
\hline 7 & 2 & $2^{4} 3^{2}(1), 2^{2} 3^{2}(1)$ & 2 & 2 & 1 & $9(1)$ & $\mathbb{Z}$ \\
\hline 8 & 2 & $2^{4} 3^{4}(2)$ & & & & & \\
\hline
\end{tabular}


TABLE 2. The cell complex, differentials, and homology for $\mathrm{GL}_{3}\left(\mathcal{O}_{-4}\right)$.

\begin{tabular}{|c||c|l||c|c|c|r||r|}
\hline$n$ & $\left|\Sigma_{n}^{*}\right|$ & $\mid$ Stab $\mid$ & $\left|\Sigma_{n}\right|$ & nnz & rank & elem. div. & $H_{n}$ \\
\hline 2 & 2 & $2^{7} 3^{1}(2)$ & 0 & 0 & 0 & & 0 \\
\hline 3 & 3 & $2^{3} 3^{1}(1), 2^{5} 3^{1}(2)$ & 0 & 0 & 0 & & 0 \\
\hline 4 & 4 & $2^{4}(1), 2^{3}(1), 2^{5}(1), 2^{7} 3^{1}(1)$ & 1 & 0 & 0 & & $\mathbb{Z}$ \\
\hline 5 & 5 & $2^{2} 3^{1}(2), 2^{3}(1), 2^{5} 3^{1}(1), 2^{5}(1)$ & 4 & 0 & 0 & & $\mathbb{Z} \oplus \mathbb{Z}_{2}$ \\
\hline 6 & 3 & $2^{2} 3^{1}(2), 2^{4}(1)$ & 3 & 10 & 3 & $1(2), 2(1)$ & 0 \\
\hline 7 & 1 & $2^{4}(1)$ & 0 & 0 & 0 & & 0 \\
\hline 8 & 1 & $2^{7} 3^{1}(1)$ & 1 & 0 & 0 & & $\mathbb{Z}$ \\
\hline
\end{tabular}

TABLE 3. The cell complex, differentials, and homology for $\mathrm{GL}_{3}\left(\mathcal{O}_{-7}\right)$.

\begin{tabular}{|c||c|l||c|c|c|c||r|}
\hline$n$ & $\left|\Sigma_{n}^{*}\right|$ & $\mid$ Stab & $\left|\Sigma_{n}\right|$ & nnz & rank & elem. div. & $H_{n}$ \\
\hline 2 & 3 & $2^{4} 3^{1}(3)$ & 0 & 0 & 0 & & 0 \\
\hline 3 & 6 & $\begin{array}{l}2^{2} 3^{1}(2), 2^{3} 3^{1}(1), 2^{2}(1), 2^{4} 3^{1}(1), \\
2^{4}(1)\end{array}$ & 0 & 0 & 0 & & 0 \\
\hline 4 & 9 & $\begin{array}{l}2^{2} 3^{1}(1), 2^{1}(1), 2^{3} 3^{1}(1), 2^{2}(2), \\
2^{4}(1), 2^{3}(3)\end{array}$ & 3 & 0 & 0 & & \\
\hline 5 & 11 & $\begin{array}{l}2^{2} 3^{1}(1), 2^{1}(2), 2^{2}(1), 2^{4} 3^{1}(3), \\
2^{1} 3^{1}(3), 2^{3}(1)\end{array}$ & 10 & 8 & 2 & $1(2)$ & $\mathbb{Z}^{2} \oplus \mathbb{Z}_{7}$ \\
\hline 6 & 8 & $2^{2}(2), 2^{1} 3^{1}(2), 2^{3}(3), 2^{1} 3^{1} 7^{1}(1)$ & 6 & 19 & 6 & $1(5), 7(1)$ & 0 \\
\hline 7 & 2 & $2^{1} 7^{1}(1), 2^{2}(1)$ & 1 & 0 & 0 & & $\mathbb{Z}_{3}$ \\
\hline 8 & 2 & $2^{4} 3^{1} 7^{1}(1), 2^{1} 3^{1} 7^{1}(1)$ & 2 & 2 & 1 & $3(1)$ & $\mathbb{Z}$ \\
\hline
\end{tabular}

TABLE 4. The cell complex, differentials, and homology for $\mathrm{GL}_{3}\left(\mathcal{O}_{-8}\right)$.

\begin{tabular}{|c||c|l||c|c|c|c||r|}
\hline$n$ & $\left|\Sigma_{n}^{*}\right|$ & $\mid$ Stab & $\left|\Sigma_{n}\right|$ & nnz & rank & elem. div. & $H_{n}$ \\
\hline 2 & 5 & $2^{2} 3^{1}(2), 2^{4} 3^{1}(2), 2^{4}(1)$ & 0 & 0 & 0 & & 0 \\
\hline 3 & 16 & $\begin{array}{l}2^{1}(2), 2^{2}(5), 2^{3} 3^{1}(1), 2^{2} 3^{1}(5), \\
2^{4} 3^{1}(1), 2^{5}(2)\end{array}$ & 2 & 0 & 0 & & 0 \\
\hline 4 & 26 & $\begin{array}{l}2^{1}(14), 2^{2}(9), 2^{3}(1), 2^{5} 3^{1}(1), \\
2^{4}(1)\end{array}$ & 16 & 12 & 2 & $1(2)$ & $\mathbb{Z} \oplus \mathbb{Z}_{2}$ \\
\hline 5 & 37 & $\begin{array}{l}2^{1}(25), 2^{2}(4), 2^{1} 3^{1}(4), 2^{3}(2), \\
2^{4} 3^{1}(2)\end{array}$ & 36 & 104 & 13 & $1(12), 2(1)$ & $\mathbb{Z}^{2} \oplus \mathbb{Z}_{2}$ \\
\hline 6 & 28 & $\begin{array}{l}2^{1}(18), 2^{2}(2), 2^{1} 3^{1}(4), 2^{3}(2), \\
2^{2} 3^{1}(2)\end{array}$ & 26 & 166 & 21 & $1(20), 2(1)$ & 0 \\
\hline 7 & 7 & $2^{1}(6), 2^{2}(1)$ & 6 & 45 & 5 & $1(5)$ & 0 \\
\hline 8 & 2 & $2^{1} 3^{1}(1), 2^{5}(1)$ & 2 & 6 & 1 & $1(1)$ & $\mathbb{Z}$ \\
\hline
\end{tabular}


ON THE COHOMOLOGY OF LINEAR GROUPS OVER IMAGINARY QUADRATIC FIELDS

TABLE 5. The cell complex, differentials, and homology for $\mathrm{GL}_{3}\left(\mathcal{O}_{-11}\right)$.

\begin{tabular}{|c||c|l||c|c|c|c||r|}
\hline$n$ & $\left|\Sigma_{n}^{*}\right|$ & $\mid$ Stab $\mid$ & $\left|\Sigma_{n}\right|$ & nnz & rank & elem. div. & $H_{n}$ \\
\hline 2 & 8 & $\begin{array}{l}2^{2} 3^{1}(2), 2^{3} 3^{1}(1), 2^{2}(2), 2^{4} 3^{1}(1), \\
2^{4}(2)\end{array}$ & 1 & 0 & 0 & & 0 \\
\hline 3 & 34 & $\begin{array}{l}2^{1}(13), 2^{2}(11), 2^{3}(2), 2^{4} 3^{1}(1), \\
2^{2} 3^{1}(4), 2^{3} 3^{1}(3)\end{array}$ & 15 & 6 & 1 & $1(1)$ & 0 \\
\hline 4 & 91 & $\begin{array}{l}2^{1}(67), 2^{2}(15), 2^{1} 3^{1}(1), 2^{3}(5), \\
2^{2} 3^{1}(1), 2^{4}(1), 2^{4} 3^{1}(1)\end{array}$ & 75 & 193 & 14 & $1(14)$ & $\mathbb{Z}$ \\
\hline 5 & 150 & $\begin{array}{l}2^{1}(124), 2^{2}(13), 2^{1} 3^{1}(7), 2^{3}(2), \\
2^{2} 3^{1}(3), 2^{4} 3^{1}(1)\end{array}$ & 147 & 700 & 60 & $1(60)$ & $\mathbb{Z}^{2} \oplus \mathbb{Z}_{4}$ \\
\hline 6 & 125 & $2^{1}(110), 2^{2}(6), 2^{1} 3^{1}(6), 2^{3}(3)$ & 122 & 859 & 85 & $1(84), 4(1)$ & 0 \\
\hline 7 & 51 & $\begin{array}{l}2^{1}(44), 2^{2}(2), 2^{1} 3^{1}(3), 2^{2} 3^{1}(1), \\
2^{4}(1)\end{array}$ & 48 & 404 & 37 & $1(37)$ & $\mathbb{Z}_{3}^{2}$ \\
\hline 8 & 12 & $2^{1}(4), 2^{1} 3^{1}(6), 2^{4}(2)$ & 12 & 88 & 11 & $1(9), 3(2)$ & $\mathbb{Z}$ \\
\hline
\end{tabular}

TABLE 6. The cell complex, differentials, and homology for $\mathrm{GL}_{3}\left(\mathcal{O}_{-15}\right)$.

\begin{tabular}{|c||c|l||c|c|c|c||r|}
\hline$n$ & $\left|\Sigma_{n}^{*}\right|$ & $\mid$ Stab $\mid$ & $\left|\Sigma_{n}\right|$ & nnz & rank & elem. div. & $H_{n}$ \\
\hline 2 & 34 & $\begin{array}{l}2^{1}(4), 2^{2}(8), 2^{1} 3^{1}(1), 2^{3}(8), \\
2^{4} 3^{1}(3), 2^{2} 3^{1}(3), 2^{4}(7)\end{array}$ & 10 & 0 & 0 & & $\mathbb{Z}$ \\
\hline 3 & 217 & $\begin{array}{l}2^{1}(102), 2^{2}(77), 2^{1} 3^{1}(2), 2^{3}(23), \\
2^{2} 3^{1}(5), 2^{4}(2), 2^{3} 3^{1}(3), 2^{4} 3^{1}(3)\end{array}$ & 128 & 175 & 9 & $1(9)$ & 0 \\
\hline 4 & 689 & $\begin{array}{l}2^{1}(546), 2^{2}(114), 2^{1} 3^{1}(1), \\
2^{3}(20), 2^{2} 3^{1}(2), 2^{4}(5), 2^{3} 3^{1}(1)\end{array}$ & 604 & 2112 & 119 & $1(119)$ & $\mathbb{Z}^{3} \oplus \mathbb{Z}_{2}^{4}$ \\
\hline 5 & 1224 & $\begin{array}{l}2^{1}(1109), 2^{2}(84), 2^{1} 3^{1}(7), \\
2^{3}(13), 2^{2} 3^{1}(5), 2^{4}(3), 2^{4} 3^{1}(3)\end{array}$ & 1185 & 6373 & 482 & $1(478), 2(4)$ & $\mathbb{Z}^{5}$ \\
\hline 6 & 1139 & $2^{1}(1081), 2^{2}(47), 2^{1} 3^{1}(7), 2^{3}(4)$ & 1102 & 7771 & 698 & $1(698)$ & 0 \\
\hline 7 & 522 & $\begin{array}{l}2^{1}(489), 2^{2}(30), 2^{1} 3^{1}(1), 2^{3}(1), \\
2^{2} 3^{1}(1)\end{array}$ & 493 & 4162 & 404 & $1(404)$ & $\mathbb{Z}_{3}^{2} \oplus \mathbb{Z}_{6}^{2} \oplus \mathbb{Z}_{12}$ \\
\hline 8 & 90 & $\begin{array}{l}2^{1}(78), 2^{2}(3), 2^{1} 3^{1}(5), 2^{3}(2), \\
2^{2} 3^{1}(2)\end{array}$ & 90 & 972 & 89 & $\begin{array}{l}1(84), 3(2), \\
6(2), 12(1)\end{array}$ & $\mathbb{Z}$ \\
\hline
\end{tabular}


TABLE 7 . The cell complex, differentials, and homology for $\mathrm{GL}_{3}\left(\mathcal{O}_{-19}\right)$.

\begin{tabular}{|c||c|l||c|c|c|c||r|}
\hline$n$ & $\left|\Sigma_{n}^{*}\right|$ & $\mid$ Stab $\mid$ & $\left|\Sigma_{n}\right|$ & nnz & rank & elem. div. & $H_{n}$ \\
\hline 2 & 43 & $\begin{array}{l}2^{1}(23), 2^{2}(10), 2^{4} 3^{1}(1), 2^{1} 3^{1}(3), \\
2^{3}(1), 2^{2} 3^{1}(2), 2^{4}(2), 2^{3} 3^{1}(1)\end{array}$ & 29 & 0 & 0 & & 0 \\
\hline 3 & 359 & $\begin{array}{l}2^{1}(304), 2^{2}(38), 2^{1} 3^{1}(3), 2^{3}(7), \\
2^{2} 3^{1}(4), 2^{3} 3^{1}(2), 2^{4} 3^{1}(1)\end{array}$ & 314 & 664 & 29 & $1(29)$ & 0 \\
\hline 4 & 1293 & $\begin{array}{l}2^{1}(1234), 2^{2}(52), 2^{3}(4), 2^{4}(1), \\
2^{3} 3^{1}(1), 2^{4} 3^{1}(1)\end{array}$ & 1255 & 5410 & 285 & $1(285)$ & $\mathbb{Z}^{2}$ \\
\hline 5 & 2347 & $\begin{array}{l}2^{1}(2287), 2^{2}(37), 2^{1} 3^{1}(13), \\
2^{3}(7), 2^{2} 3^{1}(2), 2^{4} 3^{1}(1)\end{array}$ & 2339 & 13596 & 968 & $1(968)$ & $\mathbb{Z}^{3} \oplus \mathbb{Z}_{2} \oplus \mathbb{Z}_{8}$ \\
\hline 6 & 2169 & $2^{1}(2137), 2^{2}(15), 2^{1} 3^{1}(10), 2^{3}(7)$ & 2164 & 15404 & 1368 & $\begin{array}{l}1(1366), \\
2(1), 8(1)\end{array}$ & 0 \\
\hline 7 & 958 & & & & & & \\
\hline 8 & 157 & $2^{1}(950), 2^{2}(6), 2^{1} 3^{1}(1), 2^{4}(1)$ & 952 & 8181 & 796 & $1(796)$ & $\mathbb{Z}_{3}^{4}$ \\
\hline
\end{tabular}

TABLE 8. The cell complex, differentials, and homology for $\mathrm{GL}_{3}\left(\mathcal{O}_{-20}\right)$.

\begin{tabular}{|c|c|c|c|c|c|c|c|}
\hline$n$ & $\left|\Sigma_{n}^{*}\right|$ & $\mid$ Stab $\mid$ & $\left|\Sigma_{n}\right|$ & $\mathrm{nnz}$ & rank & elem. div. & $H_{n}$ \\
\hline 2 & 69 & $\begin{array}{l}2^{1}(21), 2^{2}(26), 2^{1} 3^{1}(4), 2^{3}(6), \\
2^{2} 3^{1}(3), 2^{4}(7), 2^{4} 3^{1}(2)\end{array}$ & 31 & 0 & 0 & & $\mathbb{Z}$ \\
\hline 3 & 538 & $\begin{array}{l}2^{1}(398), 2^{2}(98), 2^{1} 3^{1}(4), 2^{3}(22) \\
2^{2} 3^{1}(7), 2^{4}(3), 2^{3} 3^{1}(4), 2^{4} 3^{1}(2)\end{array}$ & 425 & 772 & 30 & $1(30)$ & $\mathbb{Z}_{2}$ \\
\hline 4 & 1895 & $\begin{array}{l}2^{1}(1721), 2^{2}(153), 2^{3}(15) \\
2^{2} 3^{1}(1), 2^{4}(4), 2^{4} 3^{1}(1)\end{array}$ & 1804 & 7464 & 395 & $1(394), 2(1)$ & $\mathbb{Z}^{4} \oplus \mathbb{Z}_{2}^{4}$ \\
\hline 5 & 3382 & $\begin{array}{l}2^{1}(3223), 2^{2}(117), 2^{1} 3^{1}(15), \\
2^{3}(15), 2^{2} 3^{1}(7), 2^{4}(4), 2^{4} 3^{1}(1)\end{array}$ & 3345 & 19167 & 1405 & $\begin{array}{l}1(1401) \\
2(4)\end{array}$ & $\mathbb{Z}^{6} \oplus \mathbb{Z}_{3}$ \\
\hline 6 & 3061 & $\begin{array}{l}2^{1}(2976), 2^{2}(61), 2^{1} 3^{1}(15), \\
2^{3}(8), 2^{2} 3^{1}(1)\end{array}$ & 3017 & 21502 & 1934 & $\begin{array}{l}1(1933), \\
3(1)\end{array}$ & 0 \\
\hline 7 & 1330 & $2^{1}(1293), 2^{2}(37)$ & 1294 & 11127 & 1083 & $1(1083)$ & $\mathbb{Z}_{3}^{2} \oplus \mathbb{Z}_{6}^{2} \oplus \mathbb{Z}_{12}$ \\
\hline 8 & 212 & $\begin{array}{l}2^{1}(202), 2^{2}(2), 2^{1} 3^{1}(5), 2^{3}(2), \\
2^{2} 3^{1}(1)\end{array}$ & 212 & 2532 & 211 & $\begin{array}{l}1(206), \\
3(2), 6(2), \\
12(1)\end{array}$ & $\mathbb{Z}$ \\
\hline
\end{tabular}


TABLE 9. The cell complex, differentials, and homology for $\mathrm{GL}_{3}\left(\mathcal{O}_{-23}\right)$.

\begin{tabular}{|c|c|c|c|c|c|c|c|}
\hline$n$ & $\left|\Sigma_{n}^{*}\right|$ & |Stab | & $\left|\Sigma_{n}\right|$ & nnz & rank & elem. div. & $H_{n}$ \\
\hline 2 & 204 & $\begin{array}{l}2^{1}(89), 2^{2}(65), 2^{1} 3^{1}(4), 2^{3}(27) \\
2^{2} 3^{1}(6), 2^{4}(10), 2^{4} 3^{1}(3)\end{array}$ & 126 & 0 & 0 & & $\mathbb{Z}^{4}$ \\
\hline 3 & 1777 & $\begin{array}{l}2^{1}(1402), 2^{2}(295), 2^{1} 3^{1}(2), \\
2^{3}(56), 2^{2} 3^{1}(11), 2^{4}(3), 2^{3} 3^{1}(5), \\
2^{4} 3^{1}(3)\end{array}$ & 1477 & 3272 & 122 & $1(122)$ & $\mathbb{Z}_{2}$ \\
\hline 4 & 6589 & $\begin{array}{l}2^{1}(6112), 2^{2}(434), 2^{3}(35) \\
2^{2} 3^{1}(2), 2^{4}(5), 2^{3} 3^{1}(1)\end{array}$ & 6285 & 26837 & 1355 & $\begin{array}{l}1(1354) \\
2(1)\end{array}$ & $\mathbb{Z}^{5} \oplus \mathbb{Z}_{2}^{10} \oplus \mathbb{Z}_{6}^{3}$ \\
\hline 5 & 12214 & $\begin{array}{l}2^{1}(11866), 2^{2}(291), 2^{1} 3^{1}(19) \\
2^{3}(23), 2^{2} 3^{1}(6), 2^{4}(6), 2^{4} 3^{1}(3)\end{array}$ & 12119 & 69891 & 4925 & $\begin{array}{l}1(4912) \\
2(10), 6(3)\end{array}$ & $\mathbb{Z}^{10} \oplus \mathbb{Z}_{2}^{5}$ \\
\hline 6 & 11627 & $\begin{array}{l}2^{1}(11461), 2^{2}(138), 2^{1} 3^{1}(16) \\
2^{3}(10), 2^{2} 3^{1}(2)\end{array}$ & 11568 & 81720 & 7184 & $\begin{array}{l}1(7179) \\
2(5)\end{array}$ & 0 \\
\hline 7 & 5303 & $\begin{array}{l}2^{1}(5250), 2^{2}(48), 2^{1} 3^{1}(2) \\
2^{2} 3^{1}(2), 2^{4}(1)\end{array}$ & 5253 & 44741 & 4384 & $1(4384)$ & $\mathbb{Z}_{12}^{2} \oplus \mathbb{Z}_{3}^{2} \oplus \mathbb{Z}_{6}^{3}$ \\
\hline 8 & 870 & $\begin{array}{l}2^{1}(853), 2^{2}(3), 2^{1} 3^{1}(10), 2^{3}(2), \\
2^{4}(2)\end{array}$ & 870 & 10464 & 869 & $\begin{array}{l}1(862), \\
12(2), 3(2), \\
6(3)\end{array}$ & $\mathbb{Z}$ \\
\hline
\end{tabular}

TABLE 10. The cell complex, differentials, and homology for $\mathrm{GL}_{3}\left(\mathcal{O}_{-24}\right)$.

\begin{tabular}{|c|c|c|c|c|c|c|c|}
\hline$n$ & $\left|\Sigma_{n}^{*}\right|$ & $\mid$ Stab | & $\left|\Sigma_{n}\right|$ & $\mathrm{nnz}$ & rank & elem. div. & $H_{n}$ \\
\hline 2 & 158 & $\begin{array}{l}2^{1}(90), 2^{2}(41), 2^{1} 3^{1}(3), 2^{3}(13), \\
2^{2} 3^{1}(1), 2^{4}(8), 2^{4} 3^{1}(2)\end{array}$ & 104 & 0 & 0 & & $\mathbb{Z}$ \\
\hline 3 & 1396 & $\begin{array}{l}2^{1}(1214), 2^{2}(142), 2^{1} 3^{1}(4) \\
2^{3}(24), 2^{2} 3^{1}(6), 2^{4}(1), 2^{3} 3^{1}(3) \\
2^{4} 3^{1}(2)\end{array}$ & 1247 & 2967 & 103 & $1(103)$ & $\mathbb{Z}_{2}^{7}$ \\
\hline 4 & 5090 & $\begin{array}{l}2^{1}(4859), 2^{2}(199), 2^{1} 3^{1}(1) \\
2^{3}(23), 2^{2} 3^{1}(1), 2^{4}(5), 2^{3} 3^{1}(1) \\
2^{4} 3^{1}(1)\end{array}$ & 4957 & 22280 & 1144 & $\begin{array}{l}1(1137), \\
2(7)\end{array}$ & $\mathbb{Z}^{5} \oplus \mathbb{Z}_{2}^{5}$ \\
\hline 5 & 9091 & $\begin{array}{l}2^{1}(8889), 2^{2}(161), 2^{1} 3^{1}(12) \\
2^{3}(20), 2^{2} 3^{1}(5), 2^{4}(3), 2^{4} 3^{1}(1)\end{array}$ & 9043 & 53385 & 3808 & $\begin{array}{l}1(3803), \\
2(5)\end{array}$ & $\mathbb{Z}^{7} \oplus \mathbb{Z}_{2}^{8} \oplus \mathbb{Z}_{4}^{2}$ \\
\hline 6 & 8319 & $\begin{array}{l}2^{1}(8187), 2^{2}(102), 2^{1} 3^{1}(13) \\
2^{3}(15), 2^{2} 3^{1}(2)\end{array}$ & 8263 & 58948 & 5228 & $\begin{array}{l}1(5218) \\
2(8), 4(2)\end{array}$ & $\mathbb{Z}_{2}$ \\
\hline 7 & 3662 & $2^{1}(3617), 2^{2}(42), 2^{3}(2), 2^{4}(1)$ & 3630 & 31020 & 3035 & $\begin{array}{l}1(3034) \\
2(1)\end{array}$ & $\mathbb{Z}_{12}^{2} \oplus \mathbb{Z}_{2}^{3} \oplus \mathbb{Z}_{6}$ \\
\hline 8 & 596 & $\begin{array}{l}2^{1}(578), 2^{2}(8), 2^{1} 3^{1}(2), 2^{3}(4), \\
2^{2} 3^{1}(2), 2^{4}(2)\end{array}$ & 596 & 7188 & 595 & $\begin{array}{l}1(589), \\
12(2), 2(3) \\
6(1)\end{array}$ & $\mathbb{Z}$ \\
\hline
\end{tabular}


TABLE 11. The cell complex, differentials, and homology for $\mathrm{GL}_{4}\left(\mathcal{O}_{-3}\right)$.

\begin{tabular}{|c|c|c|c|c|c|c|c|}
\hline$n$ & $\left|\Sigma_{n}^{*}\right|$ & | Stab | & $\left|\Sigma_{n}\right|$ & $\mathrm{nnz}$ & rank & elem. div. & $H_{n}$ \\
\hline 3 & 2 & $2^{4} 3^{5}(1), 2^{7} 3^{5}(1)$ & 0 & 0 & 0 & & 0 \\
\hline 4 & 5 & $\begin{array}{l}2^{4} 3^{2}(1), 2^{4} 3^{2} 5^{1}(1), 2^{5} 3^{3}(1) \\
2^{5} 3^{4}(1), 2^{3} 3^{4}(1)\end{array}$ & 0 & 0 & 0 & & 0 \\
\hline 5 & 12 & $\begin{array}{l}2^{2} 3^{2}(2), 2^{3} 3^{2}(1), 2^{2} 3^{3}(1) \\
2^{3} 3^{1}(1), 2^{5} 3^{4}(1), 2^{3} 3^{3}(1) \\
2^{4} 3^{1}(2), 2^{5} 3^{2}(2), 2^{6} 3^{4}(1)\end{array}$ & 0 & 0 & 0 & & 0 \\
\hline 6 & 34 & $\begin{array}{l}2^{5} 3^{4}(1), 2^{1} 3^{1}(1), 2^{4} 3^{1}(2) \\
2^{2} 3^{1}(8), 2^{5} 3^{3}(1), 2^{1} 3^{2}(2) \\
2^{3} 3^{4}(1), 2^{3} 3^{1}(4), 2^{5} 3^{2}(1) \\
2^{3} 3^{3}(1), 2^{4} 3^{2}(2), 2^{2} 3^{3}(4) \\
2^{2} 3^{2}(6)\end{array}$ & 8 & 0 & 0 & & $\mathbb{Z}$ \\
\hline 7 & 82 & $\begin{array}{l}2^{1} 3^{1}(21), 2^{5} 3^{1}(1), 2^{2} 3^{1}(23) \\
2^{1} 3^{2}(7), 2^{2} 3^{3}(3), 2^{3} 3^{1}(5) \\
2^{3} 3^{4}(1), 2^{2} 3^{2}(8), 2^{3} 3^{3}(2) \\
2^{7} 3^{4}(1), 2^{4} 3^{1}(4), 2^{1} 3^{3}(2) \\
2^{4} 3^{2}(1), 2^{3} 3^{2}(3)\end{array}$ & 50 & 58 & 7 & $1(7)$ & $\mathbb{Z}^{2} \oplus \mathbb{Z}_{2}$ \\
\hline 8 & 166 & $\begin{array}{l}2^{3} 3^{3}(2), 2^{1} 3^{1}(88), 2^{4} 3^{3}(1) \\
2^{2} 3^{1}(36), 2^{1} 3^{2}(13), 2^{5} 3^{3}(1) \\
2^{3} 3^{1}(5), 2^{2} 3^{2}(7), 2^{4} 3^{1}(2) \\
2^{1} 3^{3}(2), 2^{3} 3^{2}(5), 2^{2} 3^{3}(3) \\
2^{2} 3^{5}(1)\end{array}$ & 129 & 604 & 41 & $1(40), 2(1)$ & $\mathbb{Z}_{9}$ \\
\hline 9 & 277 & $\begin{array}{l}2^{1} 3^{1}(191), 2^{5} 3^{2}(1), 2^{2} 3^{1}(34) \\
2^{1} 3^{2}(17), 2^{5} 3^{5}(2), 2^{3} 3^{1}(6) \\
2^{1} 3^{1} 5^{1}(1), 2^{2} 3^{2}(9), 2^{1} 3^{3}(2) \\
2^{3} 3^{2}(3), 2^{5} 3^{1}(1), 2^{2} 3^{3}(7) \\
2^{7} 3^{3}(1), 2^{2} 3^{5}(1), 2^{4} 3^{2} 5^{1}(1)\end{array}$ & 228 & 1616 & 88 & $1(87), 9(1)$ & $\mathbb{Z} \oplus \mathbb{Z}_{24}$ \\
\hline 10 & 324 & $\begin{array}{l}2^{1} 3^{1}(246), 2^{2} 3^{1}(35), 2^{1} 3^{2}(16) \\
2^{3} 3^{1}(7), 2^{2} 3^{2}(9), 2^{1} 3^{3}(2) \\
2^{4} 3^{3}(1), 2^{2} 3^{3}(5), 2^{3} 3^{3}(2) \\
2^{5} 3^{2}(1)\end{array}$ & 286 & 2531 & 139 & $\begin{array}{l}1(138), \\
24(1)\end{array}$ & $\mathbb{Z} \oplus \mathbb{Z}_{2}^{4}$ \\
\hline 11 & 259 & $\begin{array}{l}2^{1} 3^{1}(200), 2^{2} 3^{1}(24), 2^{1} 3^{2}(11) \\
2^{3} 3^{1}(6), 2^{2} 3^{2}(9), 2^{2} 3^{4}(1) \\
2^{4} 3^{1}(1), 2^{1} 3^{3}(1), 2^{2} 3^{3}(2) \\
2^{4} 3^{2}(2), 2^{4} 3^{3}(2)\end{array}$ & 237 & 2283 & 146 & $1(142), 2(4)$ & $\mathbb{Z}_{2}^{2} \oplus \mathbb{Z}_{6}$ \\
\hline 12 & 142 & $\begin{array}{l}2^{1} 3^{1}(91), 2^{2} 3^{1}(20), 2^{1} 3^{2}(9) \\
2^{3} 3^{1}(5), 2^{2} 3^{2}(11), 2^{1} 3^{3}(4) \\
2^{2} 3^{3}(1), 2^{4} 3^{2}(1)\end{array}$ & 122 & 1252 & 91 & $\begin{array}{l}1(88), 2(2), \\
6(1)\end{array}$ & $\mathbb{Z} \oplus \mathbb{Z}_{3} \oplus \mathbb{Z}_{12}$ \\
\hline 13 & 48 & $\begin{array}{l}2^{1} 3^{3}(1), 2^{3} 3^{3}(1), 2^{1} 3^{1}(22) \\
2^{2} 3^{1}(6), 2^{1} 3^{2}(7), 2^{3} 3^{1}(2) \\
2^{2} 3^{2}(8), 2^{4} 3^{1}(1)\end{array}$ & 36 & 369 & 30 & $\begin{array}{l}1(28), 3(1), \\
12(1)\end{array}$ & $\mathbb{Z}_{15}$ \\
\hline 14 & 15 & $\begin{array}{l}2^{1} 3^{2}(2), 2^{2} 3^{2}(2), 2^{1} 3^{3}(1) \\
2^{2} 3^{3}(3), 2^{1} 3^{1}(1), 2^{4} 3^{2} 5^{1}(1) \\
2^{3} 3^{1}(1), 2^{2} 3^{1}(2), 2^{1} 3^{1} 5^{1}(1) \\
2^{6} 3^{2}(1)\end{array}$ & 10 & 51 & 6 & $1(5), 15(1)$ & $\mathbb{Z}_{12} \oplus \mathbb{Z}_{288}$ \\
\hline 15 & 5 & $\begin{array}{l}2^{6} 3^{3}(1), 2^{7} 3^{5} 5^{1}(1), 2^{4} 3^{5}(1) \\
2^{2} 3^{3}(2)\end{array}$ & 5 & 16 & 4 & $\begin{array}{l}1(2), 12(1) \\
288(1)\end{array}$ & $\mathbb{Z}$ \\
\hline
\end{tabular}


ON THE COHOMOLOGY OF LINEAR GROUPS OVER IMAGINARY QUADRATIC FIELDS

TABLE 12. The cell complex, differentials, and homology for $\mathrm{GL}_{4}\left(\mathcal{O}_{-4}\right)$.

\begin{tabular}{|c|c|c|c|c|c|c|c|}
\hline$n$ & $\left|\Sigma_{n}^{*}\right|$ & $\mid$ Stab | & $\left|\Sigma_{n}\right|$ & $\mathrm{nnz}$ & rank & elem. div. & $H_{n}$ \\
\hline 3 & 4 & $2^{11} 3^{1}(2), 2^{9} 3^{1}(1), 2^{7} 3^{1}(1)$ & 0 & 0 & 0 & & 0 \\
\hline 4 & 10 & $\begin{array}{l}2^{3} 3^{1}(1), 2^{8}(1), 2^{4} 3^{1}(1) \\
2^{5} 3^{1} 5^{1}(1), 2^{7}(1), 2^{5} 3^{1}(2) \\
2^{8} 3^{1}(1), 2^{7} 3^{1}(1), 2^{5}(1)\end{array}$ & 0 & 0 & 0 & & 0 \\
\hline 5 & 33 & $\begin{array}{l}2^{10} 3^{1}(1), 2^{2}(1), 2^{7} 3^{2}(1), 2^{6} 3^{1}(1) \\
2^{3}(6), 2^{4} 3^{1}(2), 2^{4}(7), 2^{7}(1) \\
2^{5} 3^{1}(2), 2^{3} 3^{1}(2), 2^{10}(1), 2^{6}(2) \\
2^{5}(6)\end{array}$ & 5 & 0 & 0 & & 0 \\
\hline 6 & 98 & $\begin{array}{l}2^{7}(1), 2^{7} 3^{1}(2), 2^{2}(26), 2^{3}(37) \\
2^{2} 3^{1}(1), 2^{4}(10), 2^{8} 3^{2}(1), 2^{3} 3^{1}(1) \\
2^{5}(9), 2^{4} 3^{1}(4), 2^{6}(1), 2^{6} 3^{1}(1) \\
2^{5} 3^{1}(4)\end{array}$ & 48 & 35 & 5 & $1(5)$ & $\mathbb{Z} \oplus \mathbb{Z}_{2}^{4}$ \\
\hline 7 & 258 & $\begin{array}{l}2^{2}(147), 2^{3}(69), 2^{2} 3^{1}(1), 2^{4}(18) \\
2^{3} 3^{1}(2), 2^{5}(10), 2^{4} 3^{1}(3), 2^{6}(2) \\
2^{5} 3^{1}(2), 2^{7}(3), 2^{11} 3^{2}(1)\end{array}$ & 189 & 682 & 42 & $1(38), 2(4)$ & $\mathbb{Z}^{2} \oplus \mathbb{Z}_{2}^{3}$ \\
\hline 8 & 501 & $\begin{array}{l}2^{2}(397), 2^{3}(67), 2^{2} 3^{1}(4), 2^{4}(18), \\
2^{3} 3^{1}(4), 2^{5}(5), 2^{4} 3^{1}(1), 2^{6}(3), \\
2^{7}(1), 2^{5} 3^{2}(1)\end{array}$ & 435 & 2972 & 145 & $1(142), 2(3)$ & $\mathbb{Z}_{2}^{2} \oplus \mathbb{Z}_{4}$ \\
\hline 9 & 704 & $\begin{array}{l}2^{2}(603), 2^{3}(58), 2^{2} 3^{1}(6), 2^{4}(15), \\
2^{2} 5^{1}(1), 2^{3} 3^{1}(7), 2^{5}(6), 2^{4} 3^{1}(2), \\
2^{6}(1), 2^{3} 3^{2}(1), 2^{7}(1), 2^{5} 3^{1} 5^{1}(1), \\
2^{8} 3^{2}(1), 2^{9} 3^{1}(1)\end{array}$ & 639 & 5928 & 290 & $\begin{array}{l}1(287) \\
2(2), 4(1)\end{array}$ & $\mathbb{Z} \oplus \mathbb{Z}_{2}^{4} \oplus \mathbb{Z}_{4}$ \\
\hline 10 & 628 & $\begin{array}{l}2^{2}(571), 2^{3}(31), 2^{2} 3^{1}(4), 2^{4}(13), \\
2^{3} 3^{1}(3), 2^{5}(1), 2^{6}(2), 2^{3} 3^{2}(1), \\
2^{7}(1), 2^{8}(1)\end{array}$ & 597 & 6701 & 348 & $\begin{array}{l}1(343) \\
2(4), 4(1)\end{array}$ & $\mathbb{Z} \oplus \mathbb{Z}_{2}^{7} \oplus \mathbb{Z}_{4}^{2} \oplus \mathbb{Z}_{8} \oplus \mathbb{Z}_{24}$ \\
\hline 11 & 369 & $\begin{array}{l}2^{2}(320), 2^{3}(25), 2^{2} 3^{1}(4), 2^{4}(12) \\
2^{3} 3^{1}(4), 2^{5}(3), 2^{6} 3^{2}(1)\end{array}$ & 346 & 4544 & 248 & $\begin{array}{l}1(237) \\
2(7), 4(2) \\
8(1), 24(1)\end{array}$ & 0 \\
\hline 12 & 130 & $\begin{array}{l}2^{2}(103), 2^{3}(9), 2^{2} 3^{1}(8), 2^{4}(3), \\
2^{3} 3^{1}(1), 2^{5}(2), 2^{2} 3^{2}(1), 2^{4} 3^{1}(1), \\
2^{6}(1), 2^{5} 3^{1}(1)\end{array}$ & 120 & 1787 & 98 & $1(98)$ & $\mathbb{Z} \oplus \mathbb{Z}_{2} \oplus \mathbb{Z}_{8}$ \\
\hline 13 & 31 & $\begin{array}{l}2^{2}(13), 2^{6} 3^{1}(1), 2^{3}(7), 2^{2} 3^{1}(4), \\
2^{4}(1), 2^{3} 3^{1}(2), 2^{6}(1), 2^{5} 3^{2}(1) \\
2^{9}(1)\end{array}$ & 22 & 337 & 21 & $\begin{array}{l}1(19), 2(1) \\
8(1)\end{array}$ & $\mathbb{Z}_{5}$ \\
\hline 14 & 7 & $\begin{array}{l}2^{3} 3^{1}(2), 2^{4}(2), 2^{5} 3^{1} 5^{1}(1) \\
2^{2} 5^{1}(1), 2^{7} 3^{1}(1)\end{array}$ & 2 & 3 & 1 & $5(1)$ & $\mathbb{Z}_{128}$ \\
\hline 15 & 2 & $2^{10} 3^{2} 5^{1}(1), 2^{11} 3^{1}(1)$ & 2 & 2 & 1 & $128(1)$ & $\mathbb{Z}$ \\
\hline
\end{tabular}




\section{REFERENCES}

[1] A. Ash, Deformation retracts with lowest possible dimension of arithmetic quotients of self-adjoint homogeneous cones, Math. Ann. 225 (1977), no. 1, 69-76.

[2] A. Ash, Small-dimensional classifying spaces for arithmetic subgroups of general linear groups, Duke Math. J. 51 (1984), no. 2, 459-468, URL: http://dx.doi.org/10.1215/S0012-7094-84-05123-8 doi:10.1215/S0012-7094-84-05123-8.

[3] A. Ash, P. E. Gunnells, and M. McConnell, Torsion in the cohomology of congruence subgroups of $\mathrm{SL}(4, \mathbb{Z})$ and Galois representations, J. Algebra 325 (2011), 404-415, URL: http://dx.doi.org/10.1016/j.jalgebra.2010.07.024 doi:10.1016/j.jalgebra.2010.07.024.

[4] A. Ash, D. Mumford, M. Rapoport, and Y.-S. Tai, Smooth compactifications of locally symmetric varieties, second ed., Cambridge Mathematical Library, Cambridge University Press, Cambridge, 2010, With the collaboration of Peter Scholze, URL: http://dx.doi.org/10.1017/CBO9780511674693 doi:10.1017/CBO9780511674693.

[5] E. Berkove, The mod-2 cohomology of the Bianchi groups, Trans. Amer. Math. Soc. 352 (2000), no. 10, 4585-4602, URL: http://dx.doi.org/10.1090/S0002-9947-00-02505-8 doi:10.1090/S0002-9947-00-025058.

[6] A. Borel and J.-P. Serre, Corners and arithmetic groups, Comment. Math. Helv. 48 (1973), 436-491, Avec un appendice: Arrondissement des variétés à coins, par A. Douady et L. Hérault.

[7] A. Borel, Some metric properties of arithmetic quotients of symmetric spaces and an extension theorem, J. Differential Geometry 6 (1972), 543-560, Collection of articles dedicated to S. S. Chern and D. C. Spencer on their sixtieth birthdays.

[8] A. Borel and L. Ji, Compactifications of symmetric and locally symmetric spaces, Mathematics: Theory \& Applications, Birkhäuser Boston, Inc., Boston, MA, 2006.

[9] W. Bosma, J. Cannon, and C. Playoust, The Magma algebra system. I. The user language, J. Symbolic Comput. 24 (1997), no. 3-4, 235-265, Computational algebra and number theory (London, 1993), URL: http://dx.doi.org/10.1006/jsco.1996.0125, doi:10.1006/jsco.1996.0125.

[10] O. Braun and R. Coulangeon, Perfect lattices over imaginary quadratic number fields, Math. Comp. 84 (2015), no. 293, 1451-1467, URL: http://dx.doi.org/10.1090/S0025-5718-2014-02891-3. doi:10.1090/S0025-5718-2014-02891-3.

[11] D. Bremner, M. Dutour Sikirić, D. V. Pasechnik, T. Rehn, and A. Schürmann, Computing symmetry groups of polyhedra, LMS J. Comput. Math. 17 (2014), 565-581.

[12] K. S. Brown, Cohomology of groups, Graduate Texts in Mathematics, vol. 87, Springer-Verlag, New York, 1994, Corrected reprint of the 1982 original.

[13] J. E. Cremona, Hyperbolic tessellations, modular symbols, and elliptic curves over complex quadratic fields, Compositio Math. 51 (1984), no. 3, 275-324, URL: http://www.numdam.org/item?id=CM_1984_51_3_275_0

[14] M. Dutour Sikirić, Polyhedral, URL: http://mathieudutour.altervista.org/Polyhedral/

[15] M. Dutour Sikirić, H. Gangl, P. E. Gunnells, J. Hanke, A. Schürmann, and D. Yasaki, On $K_{4}$ of the Gaussian and Eisenstein integers, preprint at arXiv:1411.0584

[16] M. Dutour Sikirić, H. Gangl, P. E. Gunnells, J. Hanke, A. Schürmann, and D. Yasaki, The Voronoi cone method for homology computation, in preparation.

[17] Dutour Sikirić, M., Cohoperfect, URL: https://sourceforge.net/projects/cohoperfect/

[18] P. Elbaz-Vincent, H. Gangl, and C. Soulé, Quelques calculs de la cohomologie de $\mathrm{GL}_{N}(\mathbb{Z})$ et de la K-théorie de $\mathbb{Z}$, C. R. Math. Acad. Sci. Paris 335 (2002), no. 4, 321-324, URL: http://dx.doi.org/10.1016/S1631-073X(02)02481-0 doi:10.1016/S1631-073X(02)02481-0.

[19] P. Elbaz-Vincent, H. Gangl, and C. Soulé, Perfect forms, K-theory and the cohomology of modular groups, Adv. Math. 245 (2013), 587-624, URL: http://dx.doi.org/10.1016/j.aim.2013.06.014 doi:10.1016/j.aim.2013.06.014.

[20] P. E. Gunnells and D. Yasaki, Modular forms and elliptic curves over the cubic field of discriminant -23, Int. J. Number Theory 9 (2013), no. 1, 53-76, URL: http://dx.doi.org/10.1142/S1793042112501242 doi:10.1142/S1793042112501242.

[21] G. Harder, A Gauss-Bonnet formula for discrete arithmetically defined groups, Ann. Sci. École Norm. Sup. (4) 4 (1971), 409-455.

[22] A. Hatcher, Algebraic topology, Cambridge University Press, Cambridge, 2002. 
[23] D. Jean-Guillaume, G. Thierry, G. Mark, G. Pascal, H. Bradford, K. Erich, S. David, T. Will, and V. Gilles, Linbox: A generic library for exact linear algebra, Proceedings of the International Congress of Mathematical Software, Springer, 2002, pp. 40-50.

[24] M. Koecher, Beiträge zu einer Reduktionstheorie in Positivitätsbereichen. I, Math. Ann. 141 (1960), $384-432$.

[25] M. McConnell, Classical projective geometry and arithmetic groups, Math. Ann. 290 (1991), no. 3, 441-462, URL: http://dx.doi.org/10.1007/BF01459253, doi:10.1007/BF01459253.

[26] K. Okuda and S. Yano, A generalization of Voronoï's theorem to algebraic lattices, J. Théor. Nombres Bordeaux 22 (2010), no. 3, 727-740, URL: http://jtnb.cedram.org/item?id=JTNB_2010__22_3_727_0

[27] W. Plesken and B. Souvignier, Computing isometries of lattices, J. Symbolic Comput. 24 (1997), no. 3-4, 327-334, Computational algebra and number theory (London, 1993), URL: http://dx.doi.org/10.1006/jsco.1996.0130 doi:10.1006/jsco.1996.0130.

[28] A. D. Rahm, The homological torsion of $\mathrm{PSL}_{2}$ of the imaginary quadratic integers, Trans. Amer. Math. Soc. 365 (2013), no. 3, 1603-1635, URL: http://dx.doi.org/10.1090/S0002-9947-2012-05690-X doi:10.1090/S0002-9947-2012-05690-X.

[29] A. D. Rahm and M. Fuchs, The integral homology of $\mathrm{PSL}_{2}$ of imaginary quadratic integers with nontrivial class group, J. Pure Appl. Algebra 215 (2011), no. 6, 1443-1472, URL: http://dx.doi.org/10.1016/j.jpaa.2010.09.005 doi:10.1016/j.jpaa.2010.09.005.

[30] I. Satake, On compactifications of the quotient spaces for arithmetically defined discontinuous groups, Ann. of Math. (2) 72 (1960), 555-580.

[31] A. Schürmann, Computational geometry of positive definite quadratic forms, University Lecture Series, vol. 48, American Mathematical Society, Providence, RI, 2009.

[32] A. Schürmann, Enumerating perfect forms, Quadratic forms-algebra, arithmetic, and geometry, Contemp. Math., vol. 493, Amer. Math. Soc., Providence, RI, 2009, pp. 359-377, URL: http://dx.doi.org/10.1090/conm/493/09679 doi:10.1090/conm/493/09679.

[33] J. Schwermer and K. Vogtmann, The integral homology of $\mathrm{SL}_{2}$ and $\mathrm{PSL}_{2}$ of Euclidean imaginary quadratic integers, Comment. Math. Helv. 58 (1983), no. 4, 573-598, URL: http://dx.doi.org/10.1007/BF02564653, doi:10.1007/BF02564653.

[34] M. H. Şengün, On the integral cohomology of Bianchi groups, Exp. Math. 20 (2011), no. 4, 487-505, URL: http://dx.doi.org/10.1080/10586458.2011.594671, doi:10.1080/10586458.2011.594671.

[35] J.-P. Serre, Groupes d'homotopie et classes de groupes abéliens, Ann. of Math. (2) 58 (1953), 258-294.

[36] M. D. Sikirić, A. Schürmann, and F. Vallentin, Classification of eight-dimensional perfect forms, Electron. Res. Announc. Amer. Math. Soc. 13 (2007), 21-32 (electronic), URL: http://dx.doi.org/10.1090/S1079-6762-07-00171-0, doi:10.1090/S1079-6762-07-00171-0.

[37] L. Solomon, The Steinberg character of a finite group with BN-pair, Theory of Finite Groups (Symposium, Harvard Univ., Cambridge, Mass., 1968), Benjamin, New York, 1969, pp. 213-221.

[38] C. Soulé, Addendum to the article: "On the torsion in $K_{*}(\mathbf{Z})$ " (Duke Math. J. 45 (1978), no. 1, 101-129) by R. Lee and R. H. Szczarba, Duke Math. J. 45 (1978), no. 1, 131-132.

[39] C. Soule, On the 3-torsion in $K_{4}(\mathbf{Z})$, Topology 39 (2000), no. 2, 259-265, URL: http://dx.doi.org/10.1016/S0040-9383(99)00006-3 doi:10.1016/S0040-9383(99)00006-3.

[40] R. E. Staffeldt, Reduction theory and $K_{3}$ of the Gaussian integers, Duke Math. J. 46 (1979), no. 4, 773-798, URL: http://projecteuclid.org/getRecord?id=euclid.dmj/1077313722

[41] W. Stein, Modular forms, a computational approach, Graduate Studies in Mathematics, vol. 79, American Mathematical Society, Providence, RI, 2007, With an appendix by Paul E. Gunnells.

[42] B. Stroustrup, The C++ Programming Language, 4 ed., Addison-Wesley, 2013.

[43] The GAP group, GAP - Groups, Algorithms, Programming, Version 4.4.6, URL: http://www.gap-system.org/

[44] K. Vogtmann, Rational homology of Bianchi groups, Math. Ann. 272 (1985), no. 3, 399-419, URL: http://dx.doi.org/10.1007/BF01455567, doi:10.1007/BF01455567.

[45] G. Voronoi, Nouvelles applications des paramètres continues à la théorie des formes quadratiques 1: Sur quelques propriétés des formes quadratiques positives parfaites, J. Reine Angew. Math 133 (1908), no. $1,97-178$.

[46] D. Yasaki, Hyperbolic tessellations associated to Bianchi groups, Algorithmic number theory, Lecture Notes in Comput. Sci., vol. 6197, Springer, Berlin, 2010, pp. 385-396. 
M. D. Sikirić, Rudjer Bosković Institute, Bijenicka 54, 10000 Zagreb, Croatia

E-mail address: mathieu.dutour@gmail.com

$U R L:$ http://mathieudutour.altervista.org/

H. Gangl, Department of Mathematical Sciences, South Road, Durham DH1 3LE, United KingDOM

E-mail address: herbert.gangl@durham.ac.uk

$U R L:$ http://maths.dur.ac.uk/ dmaOhg/

P. E. Gunnells, Department of Mathematics and Statistics, LGRT 1115L, University of MasSACHUSETts, AmHerst, MA 01003, USA

E-mail address: gunnells@math.umass.edu

$U R L:$ https://www.math.umass.edu/ gunnells/

J. Hanke, Princeton, NJ 08542, USA

E-mail address: jonhanke@gmail.com

$U R L:$ http://www.jonhanke.com

A. Schürmann, Universität Rostock, Institute of Mathematics, 18051 Rostock, Germany

E-mail address: achill.schuermann@uni-rostock.de

$U R L:$ http://www.geometrie.uni-rostock.de/

D. Yasaki, Department of Mathematics and Statistics, University of North Carolina at GreensBoro, Greensboro, NC 27412, USA

E-mail address: d_yasaki@uncg.edu

$U R L:$ http://www.uncg.edu/ d_yasaki/ 